\title{
Multiscale modelling of heat and mass transfer in fractured media for enhanced geothermal systems applications
}

DOI:

10.1016/j.apm.2018.10.025

\section{Document Version}

Accepted author manuscript

Link to publication record in Manchester Research Explorer

\section{Citation for published version (APA):}

Vasilyeva, M., Babaei, M., Chung, E. T., \& Spiridonov, D. (2019). Multiscale modelling of heat and mass transfer in fractured media for enhanced geothermal systems applications. Applied Mathematical Modelling.

https://doi.org/10.1016/j.apm.2018.10.025

\section{Published in:}

Applied Mathematical Modelling

\section{Citing this paper}

Please note that where the full-text provided on Manchester Research Explorer is the Author Accepted Manuscript or Proof version this may differ from the final Published version. If citing, it is advised that you check and use the publisher's definitive version.

\section{General rights}

Copyright and moral rights for the publications made accessible in the Research Explorer are retained by the authors and/or other copyright owners and it is a condition of accessing publications that users recognise and abide by the legal requirements associated with these rights.

\section{Takedown policy}

If you believe that this document breaches copyright please refer to the University of Manchester's Takedown Procedures [http://man.ac.uk/04Y6Bo] or contact uml.scholarlycommunications@manchester.ac.uk providing relevant details, so we can investigate your claim.

\section{OPEN ACCESS}




\section{Accepted Manuscript}

Multiscale modelling of heat and mass transfer in fractured media for enhanced geothermal systems applications

Maria Vasilyeva, Masoud Babaei, Eric T. Chung, Denis Spiridonov

PII:

DOI:

Reference:

To appear in:

Received date:

Revised date:

Accepted date:
S0307-904X(18)30517-1

https://doi.org/10.1016/j.apm.2018.10.025

APM 12513

Applied Mathematical Modelling

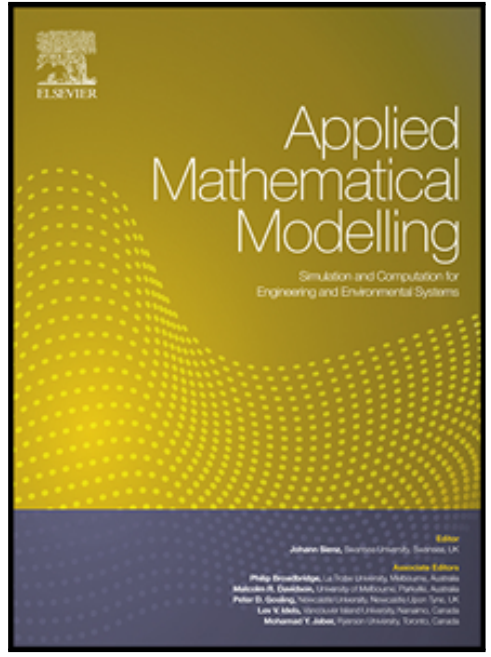

Please cite this article as: Maria Vasilyeva, Masoud Babaei, Eric T. Chung, Denis Spiridonov, Multiscale modelling of heat and mass transfer in fractured media for enhanced geothermal systems applications, Applied Mathematical Modelling (2018), doi: https://doi.org/10.1016/j.apm.2018.10.025

This is a PDF file of an unedited manuscript that has been accepted for publication. As a service to our customers we are providing this early version of the manuscript. The manuscript will undergo copyediting, typesetting, and review of the resulting proof before it is published in its final form. Please note that during the production process errors may be discovered which could affect the content, and all legal disclaimers that apply to the journal pertain. 


\section{Highlights}

- Multiscale modelling of heat and mass transfer in complex 2D and 3D fractured media

- Extension of Generalized Multiscale Finite Element Method (GMsFEM) to include heat transfer equation

- Accurate fluid pressure and temperature solution compared to fine scale

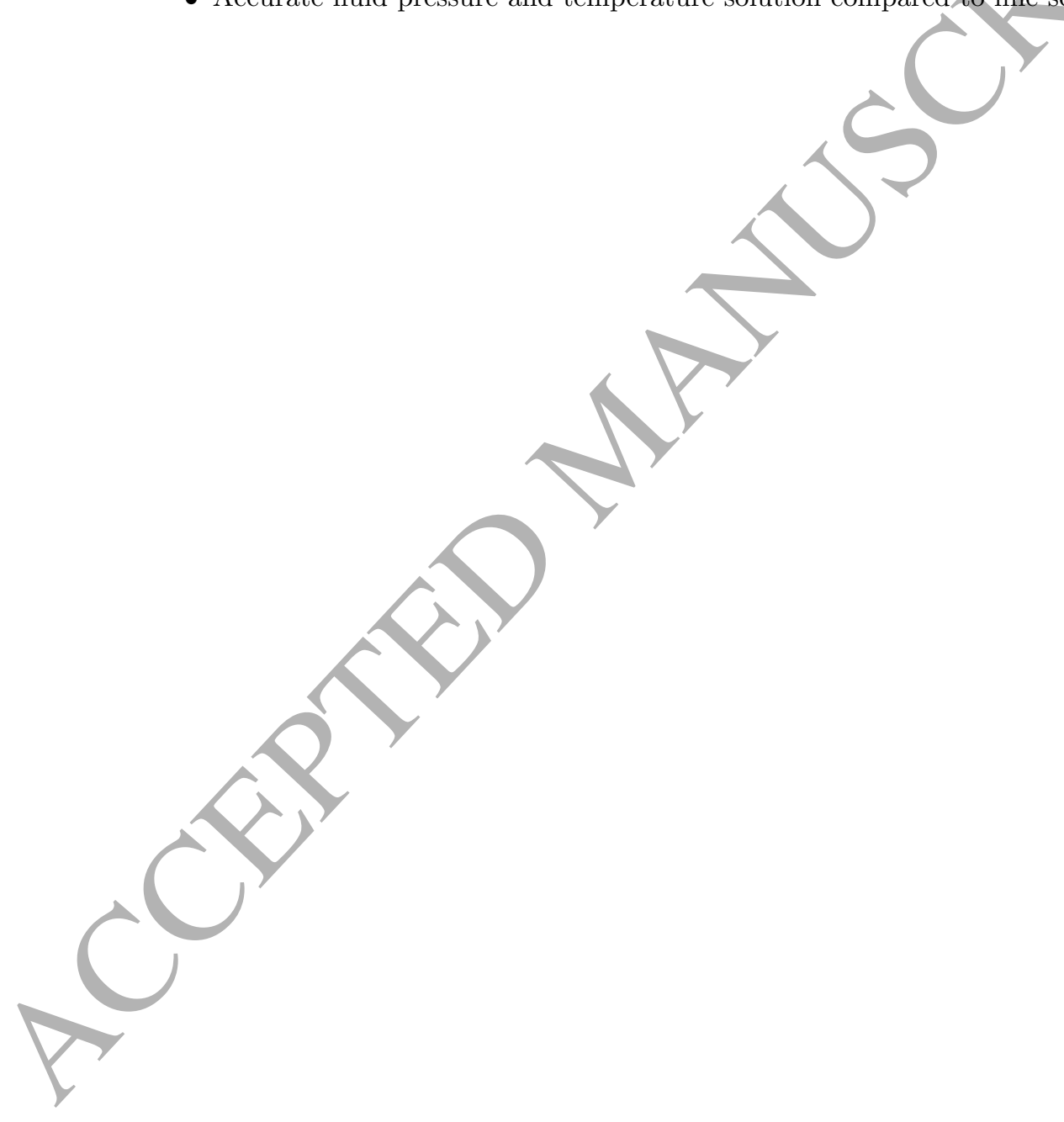




\section{Multiscale modelling of heat and mass transfer in fractured media for enhanced geothermal systems applications}

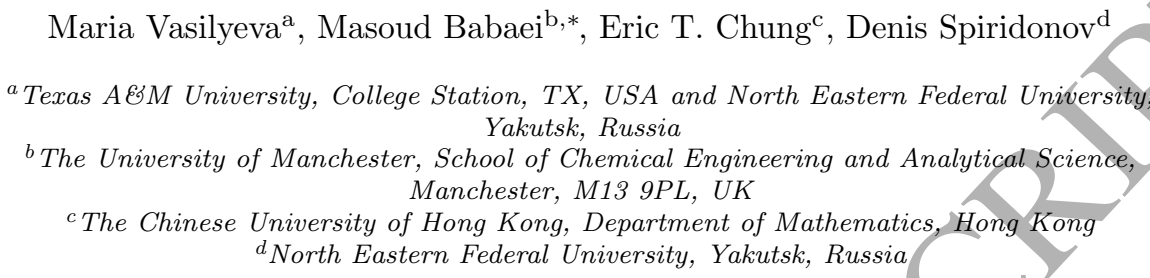

\section{Abstract}

In this work, heat and mass transfer in a hypothetical Enhanced Geothermal System with complex fracture network is considered. Fracture networks have complex geometries, exist in the multiple scales and have a significant impact on the heat and mass transfer processes. Predictive capacity of numerical models for EGS operations rely directly on how accurately the heat and mass transfer in fractures and their surrounding matrix are resolved. For numerical solution, we generate a fine grid model using finite element approximation. The fine grid explicitly resolves the fractures, however, simulation of the process leads to computationally prohibitive simulations. To reduce dimension of the system of equations, we further expand Generalized Multiscale Finite Element Method (GMsEEM) to include heat transfer equation. Multiscale basis functions for the coarse grid approximation of the equations are constructed and accurate solution fluid pressure and temperature are obtained for two and three-dimensional model problems. To the best knowledge of authors, there are only few application of multiscale methods for geothermal heat recovery operations. Therefore the developed GMsFEM for EGS applications will provide a platform to develop

\footnotetext{
* Corresponding author

Email address: masoud.babaei@manchester.ac.uk (Masoud Babaei)
} 
predictive tools for fully coupled thermo-hydro-mechanical-chemical (THMC) processes.

Keywords: Enhanced Geothermal Systems (EGS), Multiscale simulation, Fractured reservoirs, Heat recovery, Heat and mass transfer, Generalized Multiscale Finite Element Method (GMsFEM),

\section{Introduction}

To alleviate the world's energy and tackle global warming, renewable energy through geothermal systems provides an attractive option. However, the development in non-volcanic and non-sedimentary subsurface zones is essential as the geothermal heat resources in shallow sediments (such as Hot Sedimentary Aquifers) are limited [1]. To this end, the most common form of geothermal energy extraction from non-volcanic systems requires injecting cold water through an injection well and extracting heated water from a production well [2]. The Enhanced Geothermal Systems (EGS), technology that can be applied in nonvolcanic areas, circulates the water between the injection and the production wells, extracting the stored geothermal energy in the reservoir at depths of 3 to $7 \mathrm{~km}[3,4]$. An illustration of EGS technology is shown in Figure 1. The technology relies on artificially enhancing the permeability of fractured reservoirs through (i) a stimulation process or creation phase and (ii) a subsequent production phase through new flow paths in the rock created from shear-failure $[5,6]$. The modeling of these two phases involve coupled thermal-hydro-mechanical and thermal-hydro-chemical, respectively. In both phases, the fractured network properties have significant impact on fluid flow and heat transfer, and should not be oversimplified or averaged out. However, most of the simulation suites rely on continuum-scale average model to predict the flow and heat transfer, and one of the largest challenges or uncertainties, is whether or not the existing continuum-scale models that use the so-called homogenized matrixfracture properties are able to capture the dynamics accurately and reliably [7]. To date, it has remained a major challenge to develop codes and computational 
methods that are adapted to the simulation of large, high-resolution domains, particularly with respect to discrete fractures and fracture networks [8].

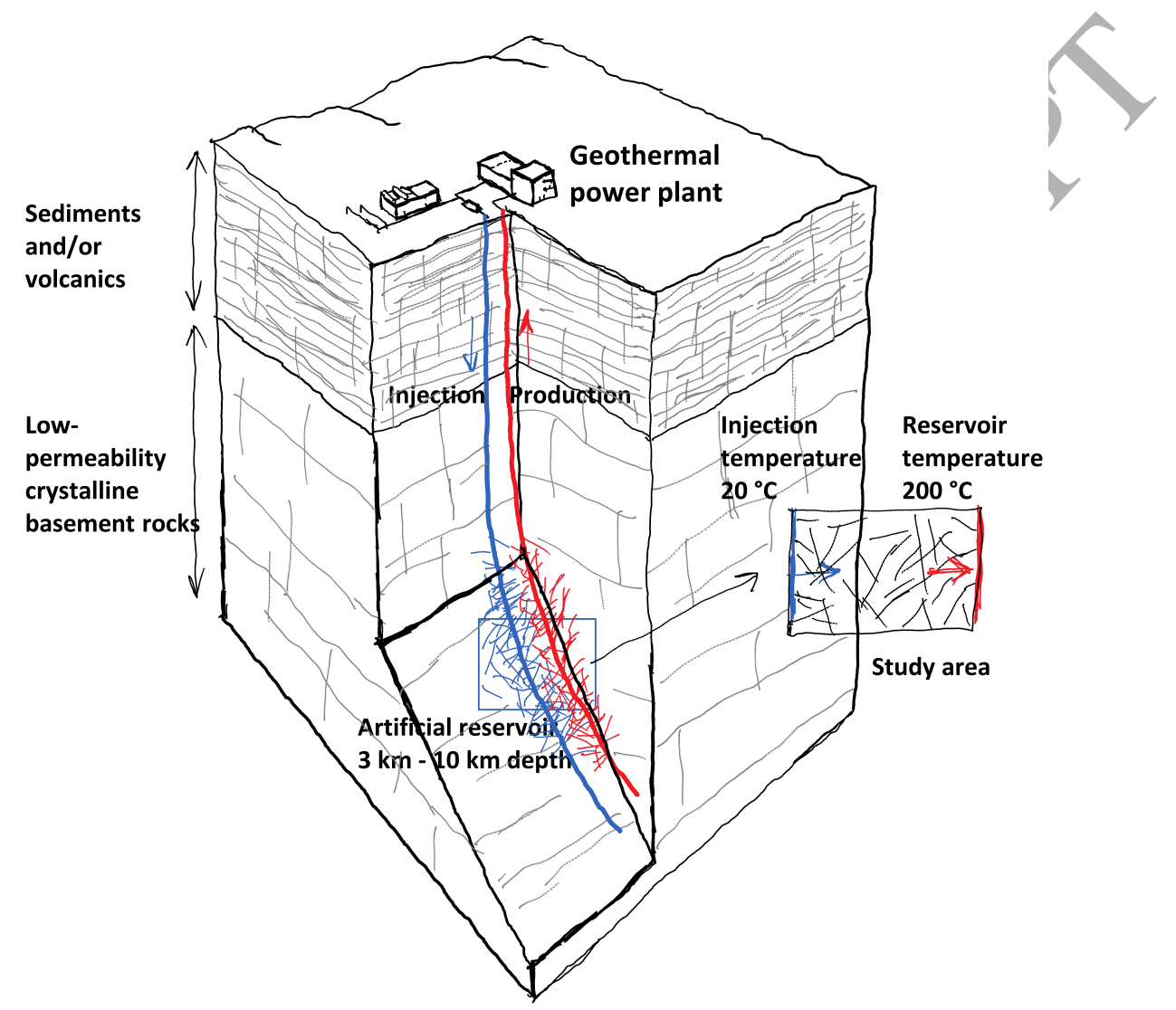

Figure 1: Schematic of a conceptual two-well EGS in hot rock, in a low-permeability crystalline basement formation. Figure is modified from Tester et al. [3]. The study area and initial and boundary conditions used in this paper are illustrated.

In order to enhance the accuracy of modelling, several multiscale methods that have been originally developed to generate effective platforms for resolving the length scales of subgrid geological heterogeneities - which crucially influence the transport dynamics - can be adapted to accommodate modelling the heat transfer as well. In their review, He and Tao [9] discussed the application 
of multiscale simulation of heat transfer and fluid flow problems. The authors provided four examples of coupled thermal processes (turbulent flow, launching process of a space craft, transport process in a proton exchange membrane fuel cell and condensation of refrigerant vapor on enhanced surface of a tube). However, heat recovery from heterogeneous/fractured media is not discussed in the review. Nissen et al. [10] have proposed a multiscale-based upscaling algorithm for geothermal heat transport with fractures represented explicitly in the computational grid. The coarse cells were constructed by merging fine-scale cells with similar flow properties and distance from the fracture network.) Xu et al. [11] proposed a simplified heat exchange model to simulate the coupled hydrothermal system for hot dry rock geothermal applications and implemented the model in an in-house geothermal simulation system based on an equivalent pipe network model. The authors showed that the proposed method is an efficient way to model heat transfer in industrial-scale enhanced geothermal systems. Geiser [12] discussed application of multiscale modelling in heat transfer in a multiple layer regime. The author used mobile-immobile partitioning of the porous media due to flow or adsorption, and incorporated mass exchange between the zones in the multiscale formulation. However, the media under study was not fractured. An advanced modelling of heat and mass transfer in deformable fractured media is presented by Karvounis and Jenny [6]. The authors developed an adaptive hierarchical fracture model for EGS, by employing a fracture hierarchy based on size. Discrete representations were used for large fractures, while the cloud of small fractures was represented by an upscaled effective permeability of the matrix. The approach simplified addition of new fractures to the existing grid without re-meshing. In [13], authors presented an upscaling methodology for geothermal heat transport in fractured porous media, where upscaling is based on different strategies for the advective term and the conductive term. The coarse scale advective term is constructed from sums of fine scale fluxes, whereas the coarse scale conductive term is constructed based on numerically computed basis functions. In the paper [14], the iterative multiscale finite volume method is presented, for both pressure and temperature 
unknowns, based on elliptic locally solved basis functions.

Above literature study indicates that the application of multiscale methods for geothermal heat recovery problems is an area of research in literature. In this paper, we consider a heat and mass transfer processes in fractured porous media. The novelty of this work is in the design and investigation of multiscale model reduction for heat and mass transfer in fractured porous media in $2 \mathrm{D}$ and 3D formulations using Generalized Multiscale Finite Element Method. For the fine grid approximation, we use Discrete Fracture Model (DFM), where fractures are resolved by grid explicitly. We note that, we consider only diffusion dominated processes and use continuous Galerkin approximation for pressure and temperature equations. We develop a multiscale solver using the Generalized Multiscale Finite Element Method (GMsFEM) [15, 16, 17. 18]. The purpose of GMsFEM is to find a representation of the solution by using a few multiscale basis functions per coarse region. In our GMsFEM, we use continuous Galerkin discretization and construct multiscale snapshot functions by solving local problems on overlapping coarse regions with various boundary conditions. We then perform local spectral decomposition to identify the basis functions corresponding to the dominant modes in the snapshot space. These local functions are multiplied by partition of unity functions to form conforming multiscale basis functions [19]. The multiscale model is adapted to an engineering application, and pave the way for more accurate modelling of coupled processes in deep subsurface models. Our underlying assumption in this work is that the EGS model can be ideally represented in a single-phase condition. Therefore the algorithm developed are not applicable to multiphase flow with phase change.

The paper is organized as follows. In Section 2, we present the mathematical model and the governing equations. A fine grid approximation using DFM is described in Section 3. In Section 4, we discuss the multiscale finite element approximation using GMsFEM adapted for EGS systems. In Section 5, we provide the numerical models description and parameters. In Section 6 we compare the accuracy of proposed method for different two-dimensional (2D) and three-dimensional (3D) models. We compare the solutions by choosing 
different numbers of multiscale basis functions. Finally, in Section 7, we conclude the paper and describe the future works.

\section{Problem formulation}

Mathematical modeling of the Enhanced Geothermal Systems in the fractured reservoirs requires the modeling of coupled fluid and heat flows. The mathematical model is described by the coupled system for temperature and pressure in the porous matrix and in the fracture network. Let $\Omega \subset \mathcal{R}^{d}(d=$ $2,3)$ be the computational domain for porous matrix and $\gamma \subset \mathcal{R}^{d-1}$ be the lower dimensional domain for fractures.

Let $p$ be the pressure and $T$ be the temperature. We assume a slightly compressible fluids, where the change of density $\rho$ and porosity $\phi$ is related to the change in temperature and pressure

$$
\begin{aligned}
& \rho(p, T)=\rho_{0}\left(1-c_{T}^{w}\left(T-T_{0}\right)+c_{p}^{w}\left(p-p_{0}\right)\right), \\
& \phi(p, T)=\phi_{0}\left(1-c_{T}^{r}\left(T-T_{0}\right)+c_{p}^{r}\left(p-p_{0}\right)\right),
\end{aligned}
$$

where $c_{T}^{w}$ and $c_{T}^{r}$ are the fluid and rock thermal expansion coefficients, and $c_{p}^{w}$ and $c_{p}^{r}$ are the fluid and rock compression coefficients.

Fluid flow in the porous matrix and fractures. For fluid flow in the porous matrix, we have a mass conservation and Darcy's law:

$$
\begin{gathered}
\frac{\partial(\rho \phi)}{\partial t}+\operatorname{div}\left(\rho q_{m}\right)=0, \quad x \in \Omega, \\
q_{m}=-\frac{\kappa_{m}}{\mu} \operatorname{grad} p_{m}, \quad x \in \Omega,
\end{gathered}
$$

where $p_{m}$ is the matrix pressure, $q_{m}$ is Darcy velocity, $\mu$ is the fluid viscosity and $\kappa_{m}$ is the permeability.

Then using Eq.(1), we can write

$$
\frac{\partial(\rho \phi)}{\partial t}=\rho \frac{\partial \phi}{\partial t}+\phi \frac{\partial \rho}{\partial t}=\left(\rho \phi_{0} c_{p}^{r}+\phi \rho_{0} c_{p}^{w}\right) \frac{\partial p_{m}}{\partial t}-\left(\rho \phi_{0} c_{T}^{r}+\phi \rho_{0} c_{T}^{w}\right) \frac{\partial T_{m}}{\partial t} .
$$

Assuming slightly compressible fluids [20]

$$
\left(\rho \phi_{0} c_{p}^{r}+\phi \rho_{0} c_{p}^{w}\right) \frac{\partial p_{m}}{\partial t} \approx \phi_{0} \rho_{0}\left(c_{p}^{r}+c_{p}^{w}\right) \frac{\partial p_{m}}{\partial t},
$$




$$
\begin{aligned}
\left(\rho \phi_{0} c_{T}^{r}+\phi \rho_{0} c_{T}^{w}\right) \frac{\partial T_{m}}{\partial t} & \approx \rho_{0} \phi_{0}\left(c_{T}^{r}+c_{T}^{w}\right) \frac{\partial T_{m}}{\partial t}, \\
\operatorname{div}\left(\rho q_{m}\right) & \approx \rho_{0} \operatorname{div} q_{m},
\end{aligned}
$$

we obtain the following equation for matrix pressure and temperature in the domain $\Omega \subset \mathcal{R}^{d}$

$$
a_{m} \frac{\partial p_{m}}{\partial t}-h_{m} \frac{\partial T_{m}}{\partial t}-\operatorname{div}\left(b_{m} \operatorname{grad} p_{m}\right)=0, \quad x \in \Omega
$$

where

$$
a_{m}=\phi_{0} c_{p}^{r}+\phi_{0} c_{p}^{w}, \quad h_{m}=\phi_{0} c_{T}^{r}+\phi_{0} c_{T}^{w}, \quad b_{m}=\frac{\kappa_{m}}{\mu} .
$$

For fluid flow in the highly permeable fractures, we consider lower dimensional model in $\gamma \subset \mathcal{R}^{d-1}$

$$
\begin{aligned}
& \frac{\partial \rho}{\partial t}+\operatorname{div}\left(\rho q_{f}\right)-\rho r_{f m}=0, \quad x \in \gamma, \\
& q_{f}=-\frac{\kappa_{f}}{\mu} \operatorname{grad} p_{f}, \quad x \in \gamma, y
\end{aligned}
$$

where $p_{f}$ is the fracture pressure, $q_{f}$ is the average velocity of fluid along the fracture plane and fracture permeability $\kappa_{f}$ can be calculated using the power law $[21,22,23,24]$.

Assuming slightly compressible fluids and using following relationship

we obtain the equation for fracture pressure:

$$
\frac{\partial \rho}{\partial t}=\rho_{0} c_{p}^{w} \frac{\partial p_{f}}{\partial t}-\rho_{0} c_{T}^{w} \frac{\partial T_{f}}{\partial t}
$$

$$
a_{f} \frac{\partial p_{f}}{\partial t}-h_{f} \frac{\partial T_{f}}{\partial t}-\operatorname{div}\left(b_{f} \operatorname{grad} p_{f}\right)-r_{f m}=0
$$

\section{where}

$$
a_{f}=c_{p}^{w}, \quad h_{f}=c_{T}^{w}, \quad b_{f}=\frac{\kappa_{f}}{\mu} .
$$

The last term in Eq.(5) $\left(r_{f m}\right)$ is the mass exchange between matrix and fracture. We assume a linear relationship between flux and pressure difference

$$
r_{f m}=\sigma \eta_{f m}\left(p_{m}-p_{f}\right)
$$


Here mass exchange occurs only on the fracture boundary and should be added to the matrix pressure equation (3)

$$
r_{m f}=\sigma \eta_{m f}\left(p_{m}-p_{f}\right)
$$

where $\eta_{f m}$ and $\eta_{m f}$ are geometric factors.

Therefore, we have following system of equations for matrix and fracture pressures

$$
\begin{array}{r}
a_{m} \frac{\partial p_{m}}{\partial t}-h_{m} \frac{\partial T_{m}}{\partial t}-\operatorname{div}\left(b_{m} \operatorname{grad} p_{m}\right)+r_{m f}=0, \quad x \in \Omega, \\
a_{f} \frac{\partial p_{f}}{\partial t}-h_{f} \frac{\partial T_{f}}{\partial t}-\operatorname{div}\left(b_{f} \operatorname{grad} p_{f}\right)-r_{f m}=0, \quad x \in \gamma .
\end{array}
$$

Heat transfer in fractured porous media. Using energy conservation law, we have the following convection-diffusion equation for heat transfer in the porous matrix

$$
(c \rho)_{m} \frac{\partial T_{m}}{\partial t}+(c \rho)_{w} \operatorname{div}\left(q_{m} T_{m}\right)-\operatorname{div}\left(\lambda_{m} \operatorname{grad} T_{m}\right)=0, \quad x \in \Omega,
$$

where $q_{m}$ is the fluid velocity in porous matrix and

$$
(c \rho)_{m}=(1-\phi)(c \rho)_{r}+\phi(c \rho)_{w}, \quad \lambda_{m}=(1-\phi) \lambda_{r}+\phi \lambda_{w} .
$$

Here $(c \rho)_{r}=c_{r} \rho_{r}(c \rho)_{w}=c_{w} \rho_{w}$, where $\rho_{r}, c_{r}, \rho_{w}, c_{w}$ denote density and specific heat of the solid and fluid phases, respectively. $\lambda_{r}$ and $\lambda_{w}$ are the thermal conductivity of the solid and fluid phases, respectively [25, 26]. In Eq. 6, we assume that the volumetric heat capacities $(c \rho)_{m}$ and $(c \rho)_{w}$ are constant and can be taken outside the partial derivatives.

For heat transfer in fractures, we consider lower dimensional problem formulation in $\gamma \subset \mathcal{R}^{d-1}$

$$
(c \rho)_{f} \frac{\partial T_{f}}{\partial t}+(c \rho)_{w} \operatorname{div}\left(q_{f} T_{f}\right)-\operatorname{div}\left(\lambda_{f} \operatorname{grad} T_{f}\right)-L_{f m}=0, \quad x \in \gamma,
$$

where $(c \rho)_{f}=c_{w} \rho_{w}$, so that $\rho_{w}$ and $c_{w}$ denote density and specific heat of fluid phase in fracture, respectively. In this equation, $\lambda_{f}=\lambda_{w}$ is thermal conductivity of fluid in fracture $[27,28,29]$. Here $q_{m}$ and $q_{f}$ are the heat flux in the porous 
matrix and fracture, and $L_{f m}$ denotes a jump of the heat flux between matrix and fracture

$$
L_{f m}=\beta \eta_{f m}\left(T_{m}-T_{f}\right)+(c \rho)_{w} \sigma \eta_{f m}\left(p_{m}-p_{f}\right) T_{m f} .
$$

We add similar source term for heat transfer equation in porous matrix and obtain following system of equations for matrix and fracture temperature

$$
\begin{gathered}
(c \rho)_{m} \frac{\partial T_{m}}{\partial t}+(c \rho)_{w} \operatorname{div}\left(q_{m} T_{m}\right)-\operatorname{div}\left(\lambda_{m} \operatorname{grad} T_{m}\right)+L_{m f}=0, \quad x \in \Omega, \\
(c \rho)_{f} \frac{\partial T_{f}}{\partial t}+(c \rho)_{w} \operatorname{div}\left(q_{f} T_{f}\right)-\operatorname{div}\left(\lambda_{f} \operatorname{grad} T_{f}\right)-L_{f m}=0, \quad x \in \gamma,
\end{gathered}
$$

where

$$
L_{m f}=\beta \eta_{m f}\left(T_{m}-T_{f}\right)+(c \rho)_{w} \sigma \eta_{m f}\left(p_{m}-p_{f}\right) T_{m f} .
$$

and $T_{m f}$ is choosen using upwind technique.

\section{Discrete problem on the fine grid}

Various numerical approaches to model fractured porous media have been developed and can be classified by the types of meshing techniques used for simulations. One approach, called discrete fracture model (DFM) is associated with the conforming discretization or explicit meshing of the fracture geometry. In another approach, called the embedded fracture model (EFM) the fractures are not resolved by grid but are considered as an overlaying continua. In EFM, matrix and fracture are viewed as two porosity types co-existing at the same spatial location, thus simple structured meshes can be used for the domain discretization $[30,31,32]$. In EFM separete meshing of the fracture networks can help to handle a dynamic fracture networks $[33,34]$. We will consider it in future works.

In this work, for approximation on the fine grid, we use conforming discretization or explicit meshing of DFM. In DFM, the fractures are located on interfaces between matrix cells $[35,36]$. Let $\mathcal{T}_{h}$ be the fine grid for the domain $\Omega, \mathcal{E}_{h}$ be the facets of the mesh $\mathcal{T}_{h}$, and $\mathcal{E}_{\gamma}$ be the fracture facets, where $\mathcal{E}_{\gamma} \subset \mathcal{E}_{h}$. 
Variational formulation. We use continuous Gakerkin finite element method with linear basis functions. Let $V=H^{1}(\Omega)$ and $V^{h} \subset V$ denote the discrete finite element space on computational mesh $\mathcal{T}_{h}$. We write the solution as

$$
p=\sum_{i=1}^{N_{f i}} p_{i} \phi_{i}, \quad T=\sum_{i=1}^{N_{f i}} T_{i} \phi_{i},
$$

where $\phi_{i}$ are the standard linear element basis functions defined on $\mathcal{T}_{h}$, and $N_{f i}$ denotes the number of nodes on the fine grid. Using mass exchange between fracture and matrix and superposition principle, we have the following variational formulation for heat and mass transfer problem in fractured porous media: find $\left(p^{n+1}, T^{n+1}\right) \in\left(V^{h}, V^{h}\right)$ such that

$$
\begin{aligned}
& \int_{\Omega} a_{m} \frac{p^{n+1}-p^{n}}{\tau} v d x-\int_{\Omega} h_{m} \frac{T^{n+1}-T^{n}}{\tau} v d x+\int_{\Omega} b_{m} \nabla p^{n+1} \cdot \nabla v d x \\
& +\sum_{\gamma_{j} \subset \mathcal{E}_{\gamma}} \int_{\gamma_{j}}\left[c_{f} \frac{p^{n+1}-p^{n}}{\tau} v_{f}-h_{f} \frac{T^{n+1}-T^{n}}{\tau} v_{f}+\kappa_{f} \nabla p^{n+1} \cdot \nabla v_{f}\right] d s=0 \\
& \int_{\Omega}\left((c \rho)_{m} \frac{T^{n+1}-T^{n}}{\tau}+(c \rho)_{w} \operatorname{div}\left(q_{m} T^{n+1}\right)\right) w d x+\int_{\Omega} \lambda_{m} \nabla T^{n+1} \cdot \nabla w d x \\
& +\sum_{\gamma_{j} \subset \mathcal{E}_{\gamma}} \int_{\gamma_{j}}\left[\left((c \rho)_{f} \frac{T^{n+1}-T^{n}}{\tau}+(c \rho)_{w} \operatorname{div}\left(q_{f} T^{n+1}\right)\right) w_{f}+\lambda_{f} \nabla T^{n+1} \cdot \nabla w_{f}\right] d s=0
\end{aligned}
$$

where $(v, w) \in\left(V^{h}, V^{h}\right)$ and $w_{f}, v_{f} \in V_{f}^{h} \subset V_{f}=H^{1}(\gamma)$. For approximation in time, we use implicit scheme with time step $\tau$, where $p^{n}$ and $T^{n}$ are the pressure and temperature from the previous time step.

We note that, in this approximation, we suppose that $p=p_{f}$ and $T=T_{f}$ on $\gamma$ and by applying superposition principle we obtain equations for $p$ and $T$ instead of mixed dimensional formulation with coupled system of equations for $\left(T, T_{f}\right)$ and $\left(p, p_{f}\right)$.

Matrix form and computational algorithm. For numerical solution of the coupled system of equations (9) - (10), similarly to the multiphase flow problems, some decoupling techniques can be used or this system can be solved 
on the fully coupled way. For example, in sequential method, the pressure equation is first solved, then temperature distribution is obtained. Moreover, the fluid properties are nonlinear and Newton method should be applied [14]. In this work, we consider constant properties of the system of equations (9) - (10) and decouple equations using sequential calculations of the pressure and temperature fields. For approximation by time, we use implicit schemes for both pressure and temperature equations. Therefore, we have following computational algorithm:

For each time step $t_{n}$ with $n=1,2, \ldots$

- Solve system of equations for pressure $p$

$$
S \frac{p^{n+1}-p^{n}}{\tau}+A p^{n+1}=B \frac{T^{n}-T^{n-1}}{\tau}
$$

where $A$ and $S$ are the stiffness and mass matrices

$$
\begin{gathered}
S=\left[s_{i j}\right]=\int_{\Omega} a_{m} \phi_{i} \phi_{j} d x+\sum_{\gamma_{j} \subset \mathcal{E}_{\gamma}} \int_{\gamma_{j}} a_{f} \phi_{i}^{f} \phi_{j}^{f} d s, \\
B=\left[b_{i j}\right]=\int_{\Omega} h_{m} \phi_{i} \phi_{j} d x+\sum_{\gamma_{j} \subset \mathcal{E}_{\gamma}} \int_{\gamma_{j}} h_{f} \phi_{i}^{f} \phi_{j}^{f} d s, \\
A=\left[a_{i j}\right]=\int_{\Omega} b_{m} \nabla \phi_{i} \cdot \nabla \phi_{j} d x+\sum_{\gamma_{j} \subset \mathcal{E}_{\gamma}} \int_{\gamma_{j}} b_{f} \nabla \phi_{i}^{f} \cdot \nabla \phi_{j}^{f} d s .
\end{gathered}
$$

- Solve system of equations for temperatures $T$

$$
M \frac{T^{n+1}-T^{n}}{\tau}+(C+D) T=0
$$

where $C$ is the approximation of the convective term, $M$ and $D$ are the stiffness and mass matrices

$$
\begin{gathered}
M=\left[m_{i j}\right]=\int_{\Omega}(c \rho)_{m} \phi_{i} \phi_{j} d x+\sum_{\gamma_{j} \subset \mathcal{E}_{\gamma}} \int_{\gamma_{j}}(c \rho)_{f} \phi_{i}^{f} \phi_{j}^{f} d s, \\
D=\left[d_{i j}\right]=\int_{\Omega} \lambda_{m} \nabla \phi_{i} \cdot \nabla \phi_{j} d x+\sum_{\gamma_{j} \subset \mathcal{E}_{\gamma}} \int_{\gamma_{j}} \lambda_{f} \nabla \phi_{i}^{f} \cdot \nabla \phi_{j}^{f} d s .
\end{gathered}
$$

and

$$
C=\left[c_{i j}\right]=\int_{\Omega}(c \rho)_{w} \operatorname{div}\left(q_{m} \phi_{i}\right) \phi_{j} d x+\sum_{\gamma_{j} \subset \mathcal{E}_{\gamma}} \int_{\gamma_{j}}(c \rho)_{w} \operatorname{div}\left(q_{f} \phi_{i}^{f}\right) \phi_{j}^{f} d s
$$


This fine-scale discretization yields matrices of the size $N_{f i} \times N_{f i}$ for pressure and temperature. We note that, another type of approximation techniques can be used on the fine grid, for example, the finite volume method or mixed finite element method. We will consider it in the future to improve fine grid discrete system properties. However it doesn't effect to the construction of the coarse grid approximation using GMsFEM.

\section{Coarse grid approximation using GMsFEM}

We consider a continuous Galerkin Generalized Multiscale Finite Element Method (GMsFEM) for coarse grid approximation [15, 37, 38, 39]. We will first construct a snapshot space, which contains some possible features of the solution. Then we perform a dimensional reduction procedure, using a spectral problem, to identify important modes, which are used as our multiscale basis functions. Let

$$
p_{m s}(x, t)=\sum_{i, k} p_{k}^{i}(t) \psi_{k}^{\omega_{i}}(x), \quad T_{m s}(x, t)=\sum_{i, k} T_{k}^{i}(t) \phi_{k}^{\omega_{i}}(x),
$$

where $\psi_{k}^{\omega_{i}}$ and $\phi_{k}^{\omega_{i}}$ are the multiscale basis functions for pressure and temperature that supported in local domain $\omega_{i}$, and the index $k$ represents the numbering of the basis functions [15, 40, 41, 42].

Following the fine grid solver, we apply decoupled solution technique for coarse grid system, where we first solve pressure equation and then find temperature field. We have following variational formulations:

- for pressure: find $p_{m s}^{n+1} \in V_{m s}$ such that

$$
s\left(\frac{p_{m s}^{n+1}-p_{m s}^{n}}{\tau}, v\right)+a\left(p_{m s}^{n+1}, v\right)=b\left(\frac{T_{m s}^{n}-T_{m s}^{n-1}}{\tau}, v\right), \quad \forall v \in V_{m s},
$$

where $V_{m s}$ is the multiscale space for pressure.

- for temperature: find $T_{m s} \in W_{m s}$ such that

$$
m\left(\frac{T_{m s}^{n+1}-T_{m s}^{n}}{\tau}, w\right)+c\left(T_{m s}^{n+1}, w\right)+d\left(T_{m s}^{n+1}, w\right)=0, \quad \forall w \in W_{m s},
$$

where $W_{m s}$ is the multiscale space for temperature. 
Here for bilinear forms, we have

$$
\begin{aligned}
& s(p, v)=\int_{\Omega} a_{m} p v d x+\sum_{\gamma_{j} \subset \mathcal{E}_{\gamma}} \int_{\gamma_{j}} a_{f} p v_{f} d s, \\
& b(T, v)=\int_{\Omega} h_{m} T v d x+\sum_{\gamma_{j} \subset \mathcal{E}_{\gamma}} \int_{\gamma_{j}} h_{f} T v_{f} d s, \\
& a(p, v)=\int_{\Omega} b_{m} \nabla p \cdot \nabla v d x+\sum_{\gamma_{j} \subset \mathcal{E}_{\gamma}} \int_{\gamma_{j}} b_{f} \nabla p \cdot \nabla v_{f} d s \\
& m(T, w)=\int_{\Omega}(c \rho)_{m} T w d x+\sum_{\gamma_{j} \subset \mathcal{E}_{\gamma}} \int_{\gamma_{j}}(c \rho)_{f} T w_{f} d s \\
& d(T, w)=\int_{\Omega} \lambda_{m} \nabla T \cdot \nabla w d x+\sum_{\gamma_{j} \subset \mathcal{E}_{\gamma}} \int_{\gamma_{j}} \lambda_{f} \nabla T \cdot \nabla w_{f} d s, \\
& c(T, w)=\int_{\Omega}(c \rho)_{w} \operatorname{div}\left(q_{m} T\right) w d x+\sum_{\gamma_{j} \subset \mathcal{E}} \int_{\gamma_{j}}(c \rho)_{w} \operatorname{div}\left(q_{f} T\right) w_{f} d s . \\
& \mathcal{T}^{H} \text { (Coarse Grid) }
\end{aligned}
$$

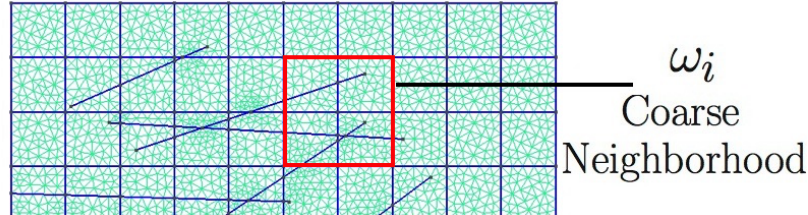

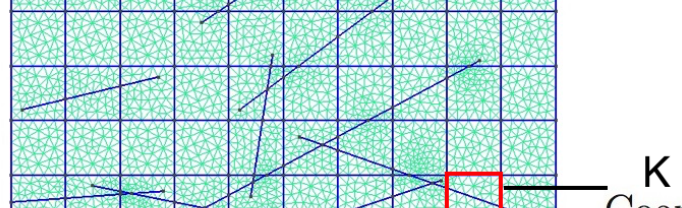

$$
\begin{aligned}
& \begin{array}{llllll}
\hline & & & & & \\
\text { Coarse } \\
\text { Element }
\end{array}
\end{aligned}
$$

Figure 2: Schematic illustration of the method. Coarse grid with local domains

In GMsFEM, we have offline and online stage. In the offline stage we construct multiscale basis functions and after that in the online stage, we solve 
our problem for any input parameters, such as right hand sides or boundary conditions [15].

Offline computations:

Step 1. Coarse grid generation.

Step 2. Construction of the snapshot space that will be used to compute an offline space.

Step 3. Construction of a "small" dimensional offline space by performing a dimension reduction in the space of local snapshots.

Next, we describe construction of the multiscale space in GMsFEM (Step 2 and 3). In this work, we construct decoupled multiscale basis functions. Coupled construction of the multiscale basis functions can provide better results and will be considered and investigated in the future works. To reduce the repetitions, we will show construction of the pressure basis functions. For the construction of the temperature basis functions, we use a similar approach. We start with the construction of the snapshot space in coarse neighborhoods (see Figure 2). The snapshot space can be the space of all fine-scale basis functions or the solutions of some local problem with various choices of boundary conditions. A general option is to use all possible boundary conditions; however, one can also use randomized boundary conditions so that one only computes a few local solutions [43]. The constructed local snapshots contain the information about local heterogeneities. We describe the details of the snapshot space construction below. Then, smaller dimensional multiscale spaces are obtained from the snapshot spaces by a dimension reduction via local spectral problems $[16,17$, 18,44 . After that, we can solve our problem in the constructed multiscale space. In this paper, we focus method on the structured coarse grids (see Figure 2). In general, this method in presented form can be extended to unstructured coarse grids, where coarse cells definite using mesh partitioner that satisfy to the load balancing property $[45,46]$. This property can be an important for fractured media with local refinements of the fine grid. We use structured grid for ease of 
implementation.

Snapshot space. For construction of the basis functions, we first define a snapshot space $V_{\text {snap }}^{\omega}$ in each local domain $\omega$. For simplicity, we omit the index $i$. The snapshot space can be the space of all fine-scale basis functions or the solutions of some local problems with various choices of the boundary conditions. We will use the latter choice in the following. In particular, we use the following problem in local domain $\omega$

$$
\begin{gathered}
a\left(\psi_{j}^{\omega, \text { snap }}, v\right)=0 \\
a(u, v)=\int_{\Omega} b_{m} \nabla u \cdot \nabla v d x+\sum_{\gamma_{j} \subset \mathcal{E}_{\gamma}} \int_{\gamma_{j}} b_{f} \nabla u_{f} \cdot \nabla v_{f} d s
\end{gathered}
$$

with Dirichlet boundary conditions

$$
\psi_{j}^{\omega, \text { snap }}=\delta_{j}^{h}(x), \quad x \in \partial \omega
$$

where $\delta_{j}^{h}(x)=\delta_{j, k}, \forall j, k \in J_{h}(\omega)$ and $J_{h}\left(\omega_{i}\right)$ denotes the fine-grid boundary node on $\partial \omega$. Therefore, we have following snapshot space for each local domain $\omega$

and

$$
V_{\text {snap }}(\omega)=\operatorname{span}\left\{\psi_{j}^{\omega, \text { snap }}: 1 \leq j \leq J_{h}\right\}
$$

$$
R_{\text {snap }}=\left[\psi_{1}^{\omega, \text { snap }}, \ldots, \psi_{J_{h}}^{\omega, \text { snap }}\right]
$$

denote a matrix representation.

Local spectral problem. In order to construct the multiscale space $V_{m s}^{\omega}$, we perform a dimension reduction of the snapshot space using an auxiliary spectral decomposition

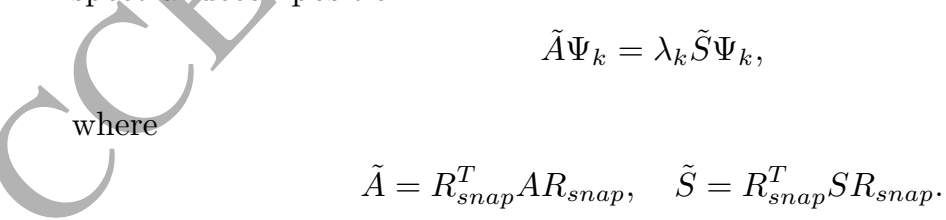

For generation of the multiscale space, we choose the smallest $M^{\omega}$ eigenvalues from Eq.(17) and form the corresponding eigenvectors in the space of snapshots by setting $\psi_{k}^{\omega, m s}=\sum_{j=1}^{J_{h}} \Psi_{k j} \psi_{j}^{\omega, s n a p}, k=1, \ldots, M^{\omega}$, where $\Psi_{k j}$ are the coordinates of the vector $\Psi_{k}$. 

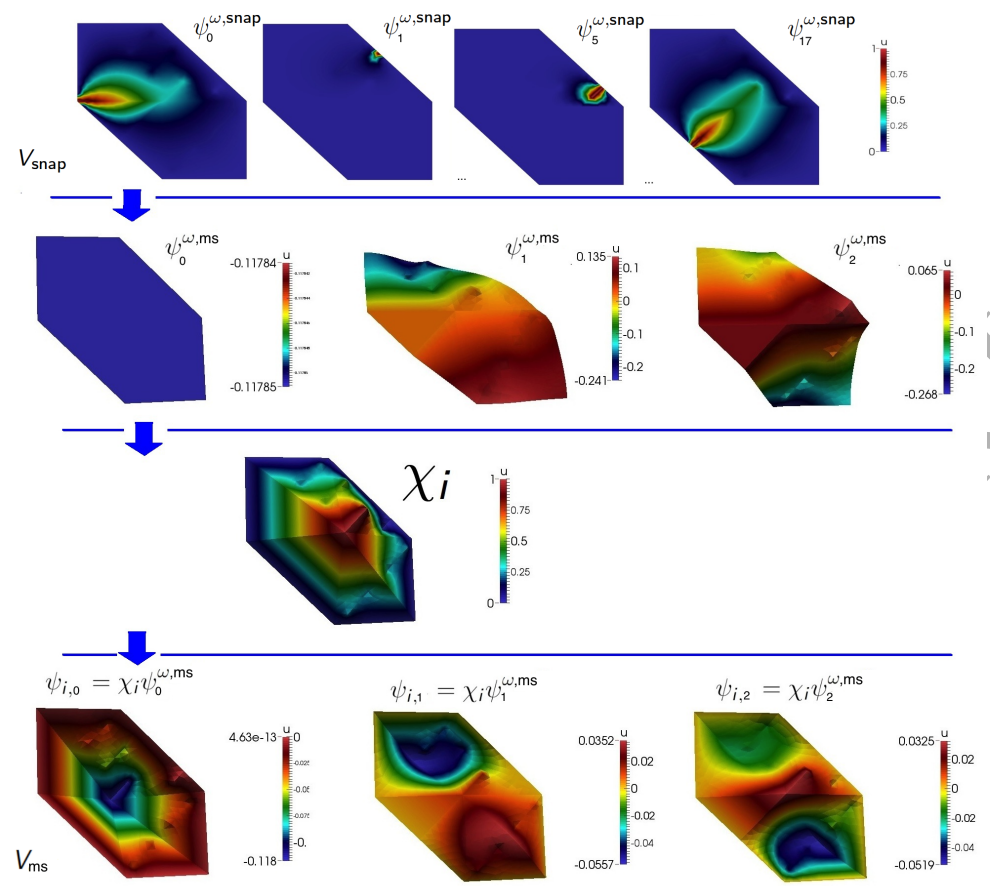

Figure 3: Construction of the multiscale basis functions for local domain $\omega$. First row: example of the snapshots constructed by solution problem (15) - (16). Second row: first three eigenvectors constructed by solution spectral problem (17). Third row: partition of unity function constructed by solution problem (18). Fourth row: multiscale basis functions as multiplication of the first three eigenvectors to partition of unity function.

Multiscale space. Let $\chi_{i}$ be the standard multiscale partition of the unity functions

$$
\begin{array}{r}
a\left(\chi_{i}, v\right)=0, \quad x \in K \\
\chi_{i}=g_{i} \quad x \in \partial K,
\end{array}
$$

for all $K \in \omega_{i}$, where $g_{i}$ is a linear function on each edge of $\partial K$.

Then, to construct the resulting basis functions, we multiply the partition of unity functions by the eigenfunctions from Eq.(17) $\psi_{i, k}=\chi_{i} \psi_{k}^{\omega_{i}, m s}(1 \leq i \leq N$ and $1 \leq k \leq M^{\omega_{i}}$, where $N$ is the number of vertices of the coarse grid). We note that, we obtain continuous basis functions due to the multiplication of 
offline eigenvectors with the initial (continuous) partition of unity $\chi_{i}$. Next, we define the continuous Galerkin spectral multiscale space as

$$
V_{m s}=\operatorname{span}\left\{\psi_{i, k}: 1 \leq i \leq N \text { and } 1 \leq k \leq M_{p}^{\omega_{i}}\right\}
$$

Construction of the multiscale space for temperature is similar and

$$
W_{m s}=\operatorname{span}\left\{\phi_{i, k}: 1 \leq i \leq N \text { and } 1 \leq k \leq M_{T}^{\omega_{i}}\right\}
$$

where $M_{T}^{\omega_{i}}$ and $M_{p}^{\omega_{i}}$ are the number of multiscale basis functions in local domain $\omega_{i}$.

In GMsFEM approach, the fracture contributions are divided in each coarse block and then coupled. Each local fracture network introduce an additional degrees of freedom for current local domain [43]. The number of dominant modes (which correspond to the very small eigenvalues) is related to the number of long and connected fracture networks, which refer to the isolated fracture network that connects the boundaries of the coarse-grid block. For each long separate fracture (by long, we mean that the fracture goes from one boundary to another), we need one additional basis function, which local spectral problem identifies. If we add more and more long fractures (that go through the entire coarse block), then the number of basis functions will increase and we need as many as the number of fractures. We remark that such spatial features are automatically captured through our local spectral problems. In contrast, many short disconnected fractures that are strictly within the coarse-grid block can be modeled with one basis functions due to design of the snapshot space. This is because their effects can be homogenized with one basis functions. The advantage of our approach is to handle general cases and the local spectral problem can automatically identify important modes.

Coarse grid system. Using constructed multiscale space for pressure and temperature, we define projection operators:

$$
\begin{aligned}
R^{T} & =\left[\psi_{1,1}, \ldots, \psi_{1, M_{p}^{\omega_{1}}}, \ldots, \psi_{N, 1}, \ldots, \psi_{N, M_{p}^{\omega_{N}}}\right] \\
P^{T} & =\left[\psi_{1,1}, \ldots, \psi_{1, M_{T}^{\omega_{1}}}, \ldots, \psi_{N, 1}, \ldots, \psi_{N, M_{T}^{\omega_{N}}}\right] .
\end{aligned}
$$


Using matrix projection operators, we have following computational algorithm: For each time step $t_{n}$ with $n=1,2, \ldots$

- Solve system of equations for pressure $p_{c}$

$$
S_{c} \frac{p_{c}^{n+1}-p_{c}^{n}}{\tau}+A_{c} p_{c}^{n+1}=B_{c} \frac{T_{c}^{n}-T_{c}^{n-1}}{\tau},
$$

- Solve system of equations for temperatures $T_{c}$

$$
M_{c} \frac{T_{c}^{n+1}-T_{c}^{n}}{\tau}+\left(C_{c}+D_{c}\right) T_{c}^{n+1}=0 .
$$

Here for the pressure equation, we have $S_{c}=R S R^{T}, A_{c}=R A R^{T}, B_{c}=$ $R B R^{T}$ and for the temperature equation, we have $M_{c}=P M P^{T}, C_{c}=P C P^{T}$ and $D_{c}=P D P^{T}$. We also note that the operator matrix may be analogously used in order to project coarse scale solutions onto the fine grid $p=R^{T} p_{c}$ and $T=P^{T} T_{c}$. Presented projection $(R$ and $P)$ and prolongation $\left(R^{T}\right.$ and $\left.P^{T}\right)$ operators can be used in iterative procedure as preconditioner [47, 14, 48, 49]. We will investigate it in future works.

\section{Numerical models and parameters}

We present numerical results for fluid flow and heat transfer in domain $\Omega$ for multiscale and fine-scale solvers. We consider four test cases:

(1) 2D domain with 15 fractures (Test Case 1),

(2) 2D domain with 100 random fractures (Test Case 2),

(3) 3D domain with 14 fractures (Test Case 3),

(4) 3D domain with 8 fractures (Test Case 4).

In Figure 4, we show computational coarse and fine grids for two-dimensional problems, where the fractures are depicted with black color and fine mesh with blue color. For Test Case 1, we consider computational domain $\Omega=[0,10]^{2} \mathrm{~m}$ and calculate multiscale solution using GMsFEM on two coarse grids: $10 \times 10$ 
and $20 \times 20$. For Test Case 2 , we consider computational domain $\Omega=[0,10]^{2}$ $\mathrm{m}$ and $20 \times 20$ coarse grid. For Test Case 3, we use coarse grid $10 \times 10 \times 5$ and computational domain $\Omega=[0,10] \times[0,10] \times[0,5]$. For Test Case 4 , we consider coarse grid $5 \times 5 \times 5$ and computational domain $\Omega=[0,10] \times[0,10] \times[0,10]$. In Figure 5, we depict coarse grid, fine grids and fracture distribution for threedimensional problems. For coarse grid approximation, we use uniform grid.
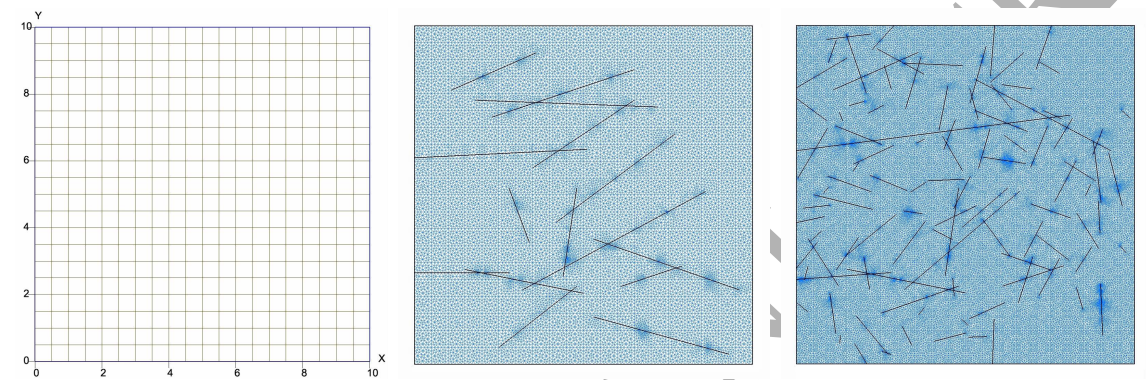

Figure 4: Computational grids. Left: uniform coarse grid $20 \times 20$ (441 vertices and 400 cells). Middle: fine grid for Test Case 1 (15534 vertices and 30666 cells). Right: fine grid for Test Case 2 (40395 vertices and 80144 cells)

We use $D O F_{f}$ (Degree of Freedom) to denote fine grid system size and $D O F_{c}$ to denote problem size of the multiscale system using GMsFEM. To compare the results, we use the weighted relative $L^{2}$ error between multiscale and fine-scale solutions

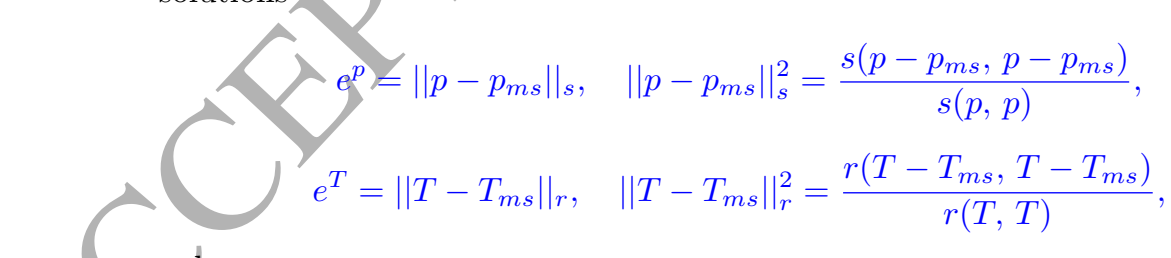

where

$$
\begin{gathered}
s(p, v)=\int_{\Omega} b_{m} p v d x+\sum_{\gamma_{j} \subset \mathcal{E}_{\gamma}} \int_{\gamma_{j}} b_{f} p v d s, \\
r(T, v)=\int_{\Omega} \lambda_{m} T v d x+\sum_{\gamma_{j} \subset \mathcal{E}_{\gamma}} \int_{\gamma_{j}} \lambda_{f} T v d s,
\end{gathered}
$$

and $p_{m s}, T_{m s}$ are the multiscale solutions and $p, T$ are the fine-scale solutions. 

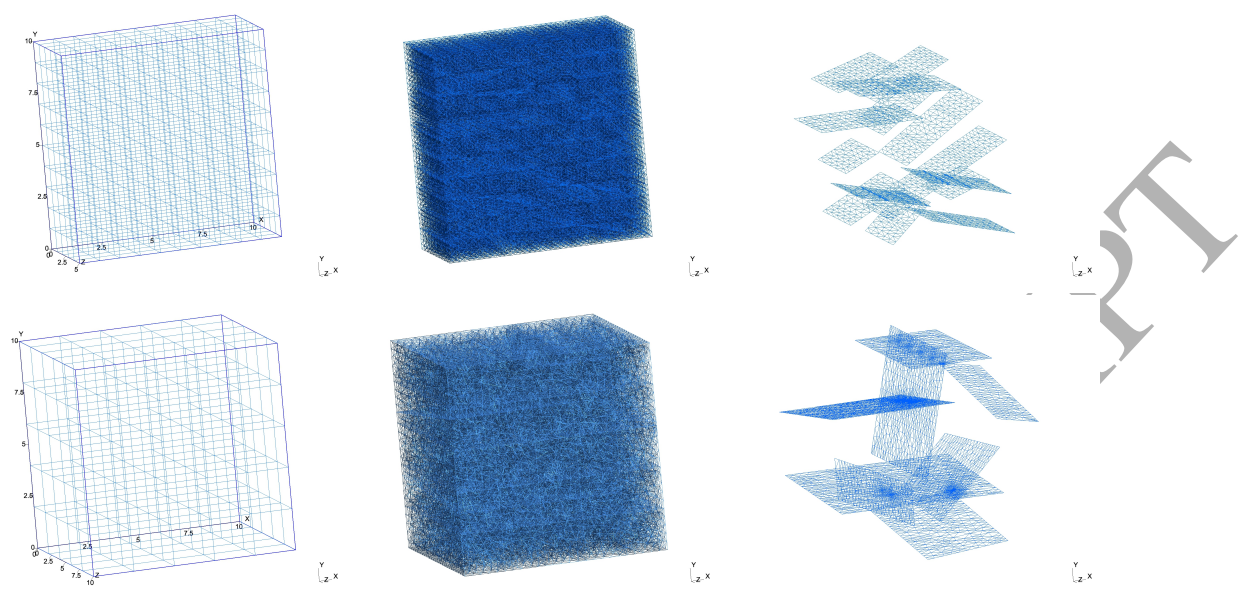

Figure 5: Computational grids for Test Case 3 (first row) and 4 (second row). First column: coarse grids. Second column: fine grids. Third column; fracture distributions.

We define two-dimensional problems for Test Cases 1 and 2 with $t_{\max }=$ 4 days and 40 time steps. We consider the following parameters for the $2 \mathrm{D}$ models: $b_{m}=0.5 \cdot 10^{-13}\left[\mathrm{~m}^{2} /(\right.$ Pa.sec $\left.)\right], b_{f}=10^{-7}\left[\mathrm{~m}^{2} /(\right.$ Pa.sec $\left.)\right], h_{m}=h_{f}=0$, $a_{m}=a_{f}=10^{-6}\left[\mathrm{~Pa}^{-1}\right],(c \rho)_{m}=(c \rho)_{f}=10^{6}\left[\mathrm{~J} /\left({ }^{\circ} \mathrm{C} \mathrm{m}{ }^{3}\right)\right], \lambda_{m}=0.1\left[\mathrm{~W} /\left({ }^{\circ} \mathrm{C} \mathrm{m}\right)\right]$, $\lambda_{f}=100.0\left[\mathrm{~W} /\left({ }^{\circ} \mathrm{C} \mathrm{m}\right)\right]$. For the boundary conditions, we set $p_{1}=5 \cdot 10^{7}[\mathrm{~Pa}]$ and $T_{1}=20\left[{ }^{\circ} \mathrm{C}\right]$ on the left boundary. On other boundaries, we set zero fluid and heat flux. We set initial pressure $p_{0}=10^{7}[\mathrm{~Pa}]$ and $T_{0}=200\left[{ }^{\circ} \mathrm{C}\right]$. In Figure 4 , we have shown the computational fine grids for two dimensional problems. For Test Case 1, we construct triangular mesh with 15534 vertices and 30666 cells. For Test Case 2, the fine grid has 40395 vertices and 80144 cells.

We consider two test cases in a three-dimensional domain for test cases 3 and 4 with $t_{\max }=4$ days and 20 time steps. We consider the following parameters for Test Case 3: $b_{m}=10^{-13}\left[\mathrm{~m}^{2} /(\right.$ Pa.sec $\left.)\right], b_{f}=10^{-6}\left[\mathrm{~m}^{2} /(\right.$ Pa.sec $\left.)\right]$, $h_{m}=h_{f}=0, a_{m}=a_{f}=10^{-6},(c \rho)_{m}=(c \rho)_{f}=10^{6}\left[\mathrm{~J} /\left({ }^{\circ} \mathrm{C} \mathrm{m} \mathrm{m}^{3}\right)\right], \lambda_{m}=$ $1.0\left[\mathrm{~W} /\left({ }^{\circ} \mathrm{C} \mathrm{m}\right)\right], \lambda_{f}=100.0\left[\mathrm{~W} /\left({ }^{\circ} \mathrm{C} \mathrm{m}\right)\right]$. For Test Case 4 , we have: $b_{m}=$ $10^{-13}\left[\mathrm{~m}^{2} /(\right.$ Pa.sec $\left.)\right], b_{f}=10^{-7}\left[\mathrm{~m}^{2} /(\right.$ Pa.sec $\left.)\right], h_{m}=h_{f}=0, a_{m}=a_{f}=10^{-6}$, $(c \rho)_{m}=(c \rho)_{f}=10^{4}\left[\mathrm{~J} /\left({ }^{\circ} \mathrm{C} \mathrm{m}{ }^{3}\right)\right], \lambda_{m}=0.1\left[\mathrm{~W} /\left({ }^{\circ} \mathrm{C} \mathrm{m}\right)\right], \lambda_{f}=100.0\left[\mathrm{~W} /\left({ }^{\circ} \mathrm{C} \mathrm{m}\right)\right]$. 
For the boundary conditions, we set $p_{1}=1.5 \cdot 10^{7}[\mathrm{~Pa}]$ and $T_{1}=20\left[{ }^{\circ} \mathrm{C}\right]$ on the left boundary for Test Case 3. For Test Case 4 , we set $p_{1}=1.1 \cdot 10^{7}[\mathrm{~Pa}]$ and $T_{1}=20\left[{ }^{\circ} \mathrm{C}\right]$ on the left boundary. On other boundaries, we set zero fluid and heat flux. We set initial pressure $p_{0}=10^{7}[\mathrm{~Pa}]$ and $T_{0}=200\left[{ }^{\circ} \mathrm{C}\right]$. Computational fine grid contains 60206 vertices for Test Case 3 and 22443 vertices for Test Case 4. For Test Case 3 , we have $10 \times 10 \times 5$ coarse grid with 726 vertices and 500 cells, and $5 \times 5 \times 5$ coarse grid with 216 vertices and 125 cells for Test Case 4 . The implementation is based on the open-source library FEniCS [50, 51].

The values used for all the parameters used in simulations are summarized in Table 1 and Table 2 .

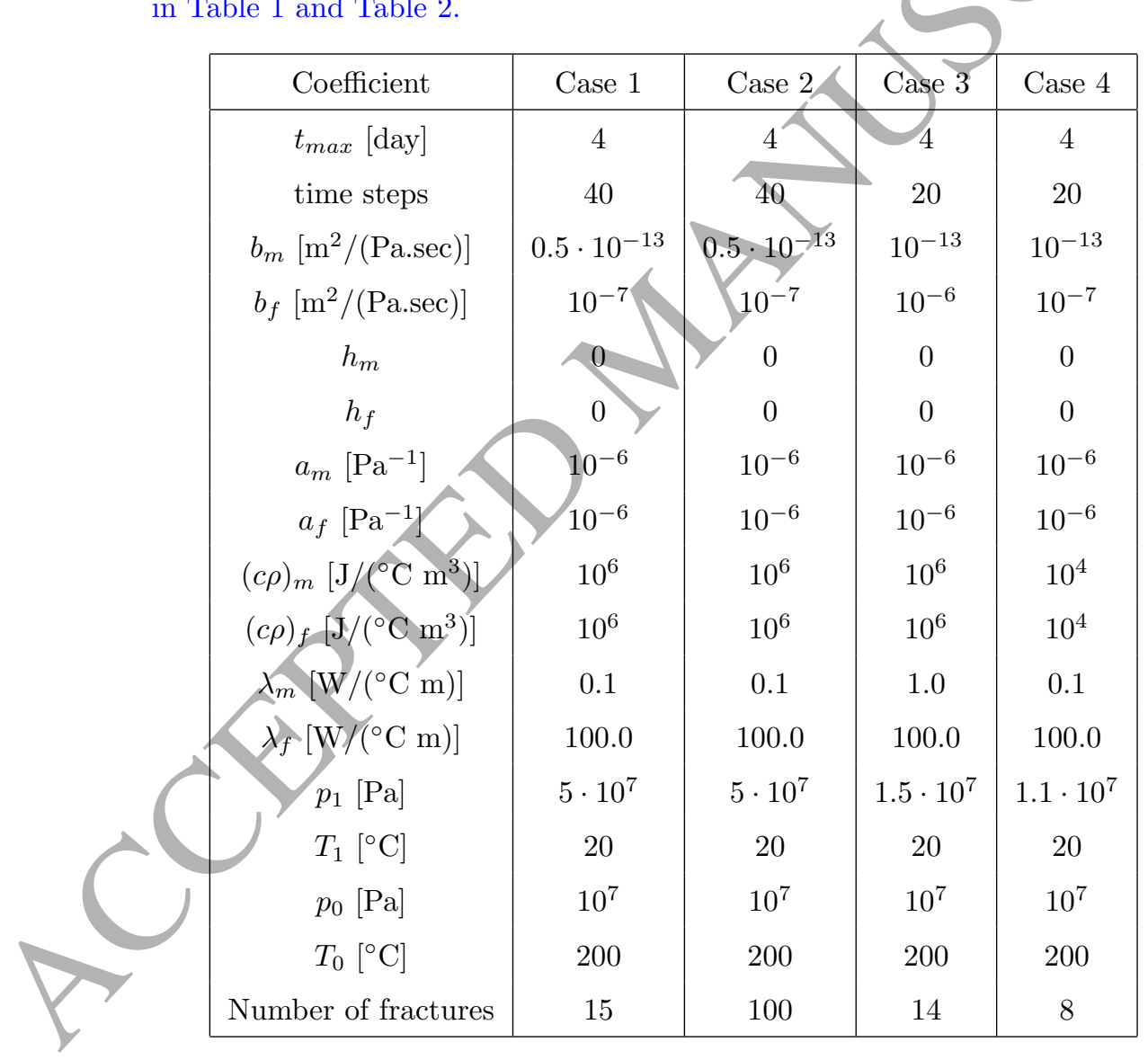

Table 1: Simulation parameters. 


\begin{tabular}{|c|c|c|c|c|}
\hline Mesh type & Case 1 & Case 2 & Case 3 & Case 4 \\
\hline Fine grid & 15534 & 40395 & 60206 & 22443 \\
Coarse grid & 441 and 121 & 441 & 726 & 216 \\
\hline
\end{tabular}

Table 2: Coarse and fine grid parameters (number of vertices).

\section{Results}

The fine-grid pressure $\left(p_{f i}\right)$ and temperature $\left(T_{f i}\right)$ distribution at times of $t_{5}, t_{20}$ and $t_{40}$ are presented in Figure 6 for Test Case 1 and in Figure 7 for Test Case 2. The figure shows that the propagation of pressure and temperature profiles are almost taking place in a same speed. This is because of assumptions made in energy equations for matrix and fracture (Eqs. 6 and 7, respectively). Realistically, there should be a delay in temperature front that our current work does not consider due to simplifying assumptions made in energy equations.

Comparison of the fine grid and multiscale solutions are presented in Figure 8 and Figure 9 at the final time. We perform computations on the $20 \times 20$ coarse grid with $M=4$ multiscale basis functions in each local domains. Fine grid system has size of $D O F_{f}=15,534$ for Test Case 1 and $D O F_{f}=40,395$ for Test Case 2. By using multiscale solver, we reduce size of system to $D O F_{c}=1,792$. At the final time, we have less than one percent of error for pressure and $1.9 \%$ for temperature for Test Case 1. For Test Case 2, we obtain 3.5\% of error for pressure and $4.2 \%$ for temperature, when we take 4 multiscale basis functions.

In Table 3, we present relative errors for Test Case 1 at final time for two coarse grids and for different number of multiscale basis functions with $M=$ 1,2,4 and 6. Relative errors for Test Case 2 are presented in Table 4 at the final time on the $20 \times 20$ coarse grid.

In Figure 10, we present relative errors for the pressure and temperature vs. time for different number of multiscale basis functions for the Test Case 2 . From the numerical results, we observe a good convergence behavior, when we take sufficient number of multiscale basis functions. 


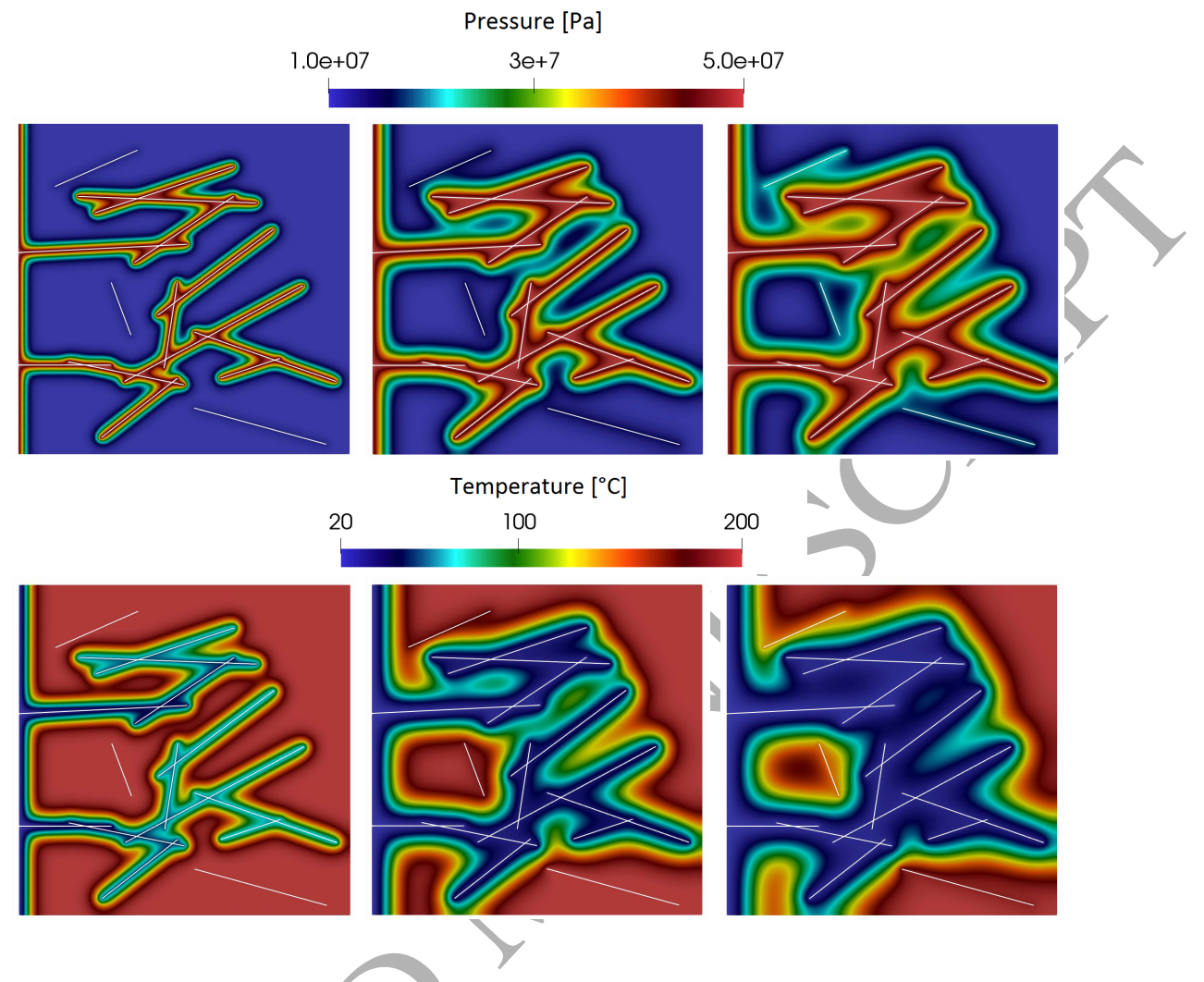

Figure 6: Fine grid solution for different times $t_{n}, n=5,20$ and 40 (from left to right) for Test Case 1. First row: pressure, $p\left(t_{n}\right)$. Second row: temperature, $T\left(t_{n}\right)$.

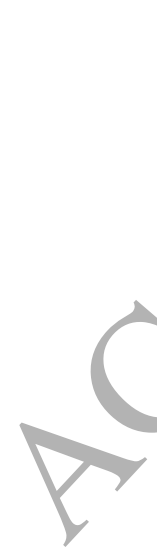

\begin{tabular}{|c|c|cc|}
\hline$M$ & $D O F_{c}$ & $e^{p}$ & $e^{T}$ \\
\hline \hline 1 & 121 & 26.233 & 55.163 \\
2 & 242 & 13.332 & 36.409 \\
4 & 484 & 2.103 & 14.743 \\
6 & 726 & 0.232 & 2.315 \\
\hline
\end{tabular}

\begin{tabular}{|c|c|cc|}
\hline$M$ & $D O F_{c}$ & $e^{p}$ & $e^{T}$ \\
\hline \hline 1 & 441 & 6.842 & 34.991 \\
2 & 882 & 0.962 & 6.834 \\
4 & 1762 & 0.240 & 1.938 \\
6 & 2646 & 0.184 & 1.700 \\
\hline
\end{tabular}

Table 3: Numerical results (relative errors $(\%)$ for the final time). Left: Coarse grid $10 \times 10$.

Right: Coarse grid $20 \times 20$. Test Case 1. Fine grid $D O F_{f}=D O F_{f}^{T}=D O F_{f}^{p}=15,534$.

We present results of the numerical simulation of three-dimensional problem for Test Case 3 in Figure 11. Relative errors are $0.458 \%$ for pressure and $3.323 \%$ 


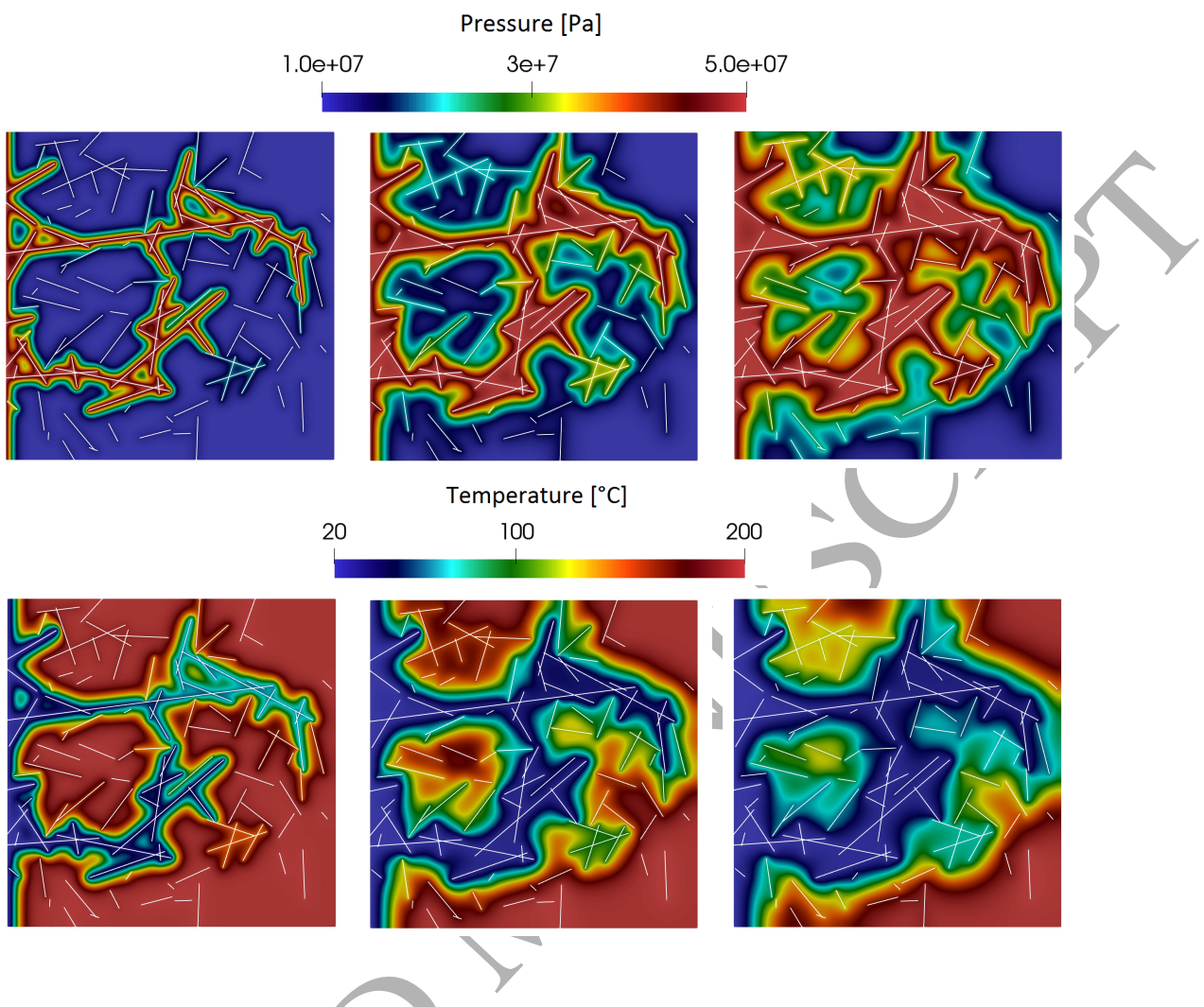

Figure 7: Fine grid solution for different times $t_{n}, n=5,20$ and 40 (from left to right) for Test Case 2. First row: pressure, $p\left(t_{n}\right)$. Second row: temperature, $T\left(t_{n}\right)$. The domain size is $10 \mathrm{~m} \times 10 \mathrm{~m}$.

\begin{tabular}{|c|c|cc|}
\hline$M$ & $D O F_{c}$ & $e^{p}$ & $e^{T}$ \\
\hline \hline 1 & 441 & 27.589 & 74.194 \\
2 & 882 & 25.962 & 33.644 \\
4 & 1762 & 3.566 & 4.273 \\
6 & 2646 & 1.876 & 2.808 \\
\hline
\end{tabular}

Table 4: Numerical results (relative errors (\%) for the final time). Coarse grid $20 \times 20$. Test Case 2. Fine grid $D O F_{f}=D O F_{f}^{T}=D O F_{f}^{p}=40,395$. 

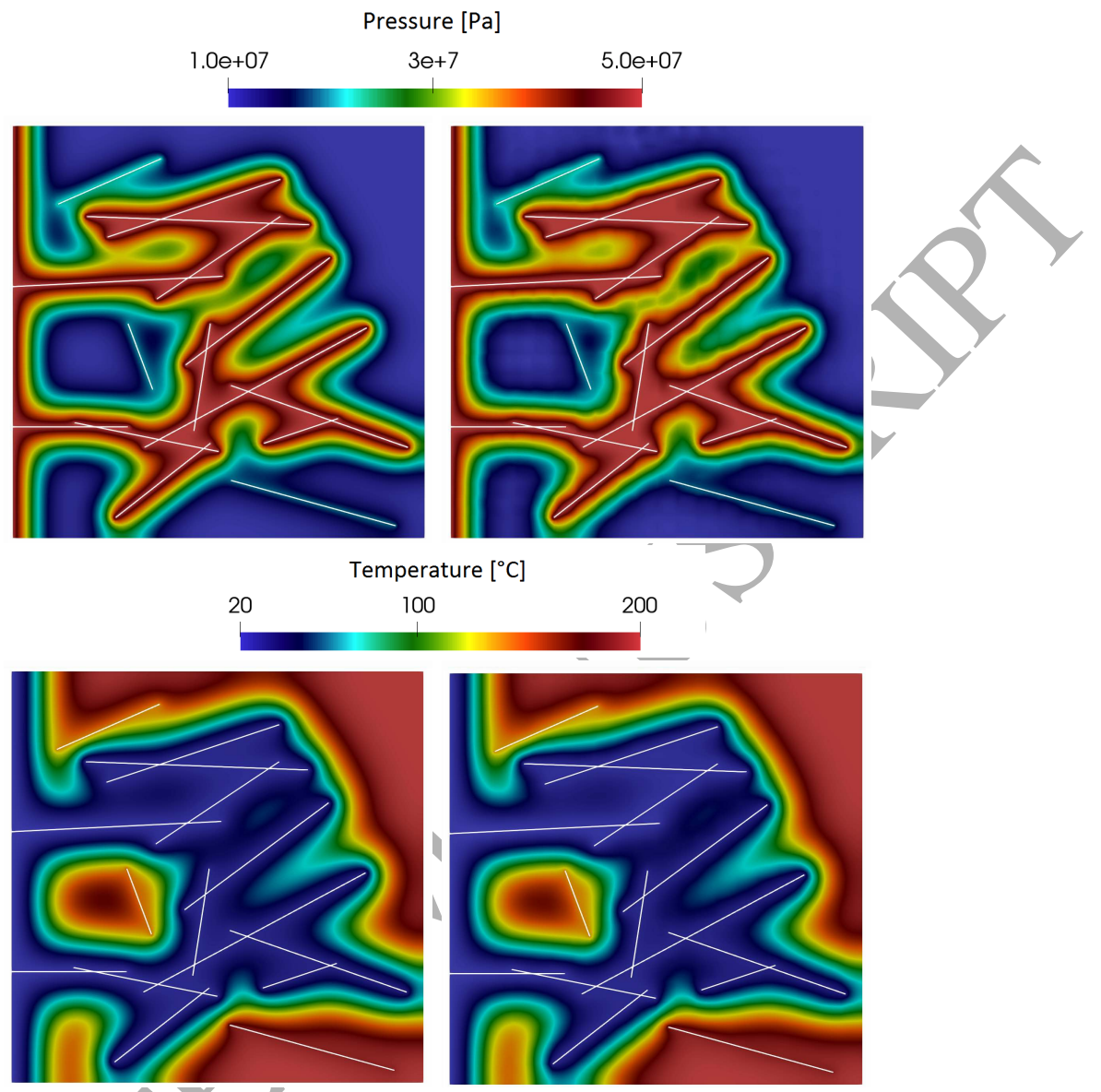

Figure 8: Fine grid and multiscale solution at final time for Test Case 1. First column: fine grid solution, $D O F_{f}=D O F_{f}^{T}=D O F_{f}^{p}=15,534$. Second column: multiscale solution, $D O F_{c}=D Q F_{c}^{T}=D O F_{c}^{p}=1762\left(11.3 \%\right.$ from $\left.D O F_{f}\right)$. First row: pressure, $p\left(t_{n}\right)$. Second row: temperature, $T\left(t_{n}\right)$. The domain size is $10 \mathrm{~m} \times 10 \mathrm{~m}$.

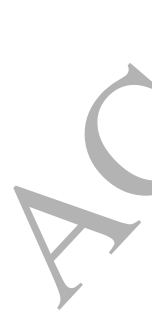

for temperature at final time. For multiscale solver, we used 6 multiscale basis functions in each local domains for pressure and same number of bases for temperature.

We present results of the numerical simulation of three-dimensional problem for Test Case 4 in Figure 12. Relative errors are $0.241 \%$ for pressure and $1.703 \%$ for temperature at final time. For multiscale solver, we used 32 multiscale basis 

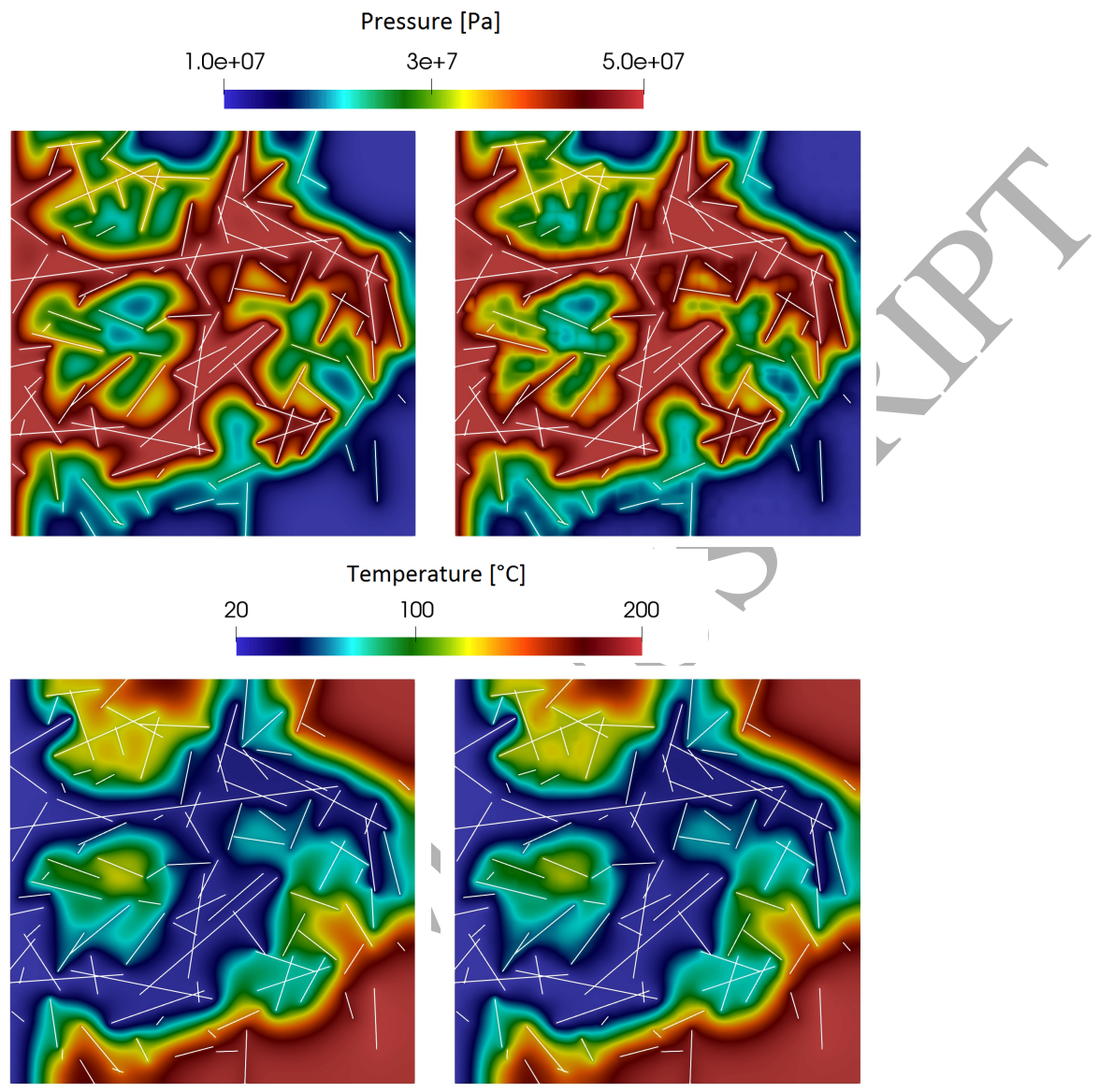

Figure 9: Fine grid and multiscale solution at final time for Test Case 2. First column: fine grid solution, $D O F_{f}=D O F_{f}^{T}=D O F_{f}^{p}=40395$. Second column: multiscale solution, $D O F_{c}=D Q F_{c}^{T} \neq D O F_{c}^{p}=1762\left(4.3 \%\right.$ from $\left.D O F_{f}\right)$. First row: pressure, $p\left(t_{n}\right)$. Second row: temperature, $T\left(t_{n}\right)$. The domain size is $10 \mathrm{~m} \times 10 \mathrm{~m}$.

functions in each local domains for pressure and same number of bases for temperature.

Next, we discuss the computational advantages of our approach and consider the online computational cost. In GMsFEM with constant coefficients, we have offline and online computational stages. In the offline stage: (a) we calculate multiscale basis functions for pressure and temperature; (b) generate projection 

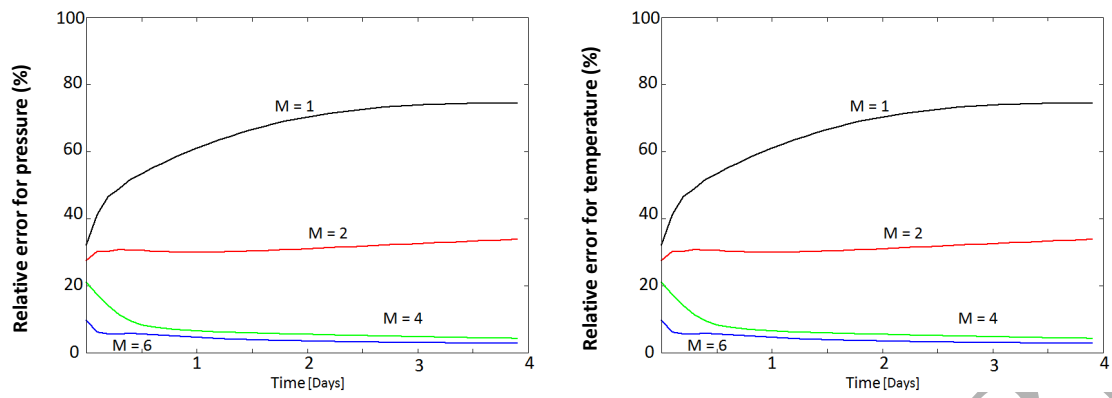

Figure 10: Relative errors vs. time for the coarse mesh $20 \times 20$ with different number of basis functions for the Test Case 2. Left: pressure, $p\left(t_{n}\right)$. Right: temperature, $T\left(t_{n}\right)$.

matrices $R$ and $P$; (c) construct coarse grid matrices $S_{c}, A_{c}, B_{c}, M_{c}$ and $D_{c}$. In the online stage: (a) we solve coarse grid system for pressure; (b) reconstruct fine grid pressure and generate convection matrix $C_{a}$ (c) solve a coarse grid system for temeperature. Main computational advantage come from fast solution of the coarse grid systems for pressure and temperature. Let $D O F_{f}^{l}$ is the size of fine-scale system $(l=p, T)$, where $D O F_{f}^{p}$ and $D O F_{f}^{T}$ are the number of degrees of freedom for pressure and for temperature fields. Then, the dimension of the fine problem is $D O F_{f}^{l} \times D O F_{f}^{l}$ for pressure and temperature for presented decoupled scheme. The coarse-scale system size is $D O F_{c}^{l}$ for pressure $(l=p)$ and the temperature problem $(l=T)$ depends on the coarse-grid size and the number of local multiscale basis functions. Assume that $N$ is the number of local domains (number of vertices on the coarse grid) and in each local domain, we have $M$ multiscale basis functions for pressure and $M$ bases for temperature. Then for presented $2 \mathrm{D}$ and $3 \mathrm{D}$ problems, we have $D O F_{c}^{p}=D O F_{c}^{T}=M \cdot N$ and $D O F_{f}^{p}=D O F_{f}^{T}=N_{f}$, where $N_{f}$ is the number of vertices on fine grid. Then, we can easily compare the computational compare the computational cost of solving coarse and fine problems. For example in Test Case 2, for coarse grid $20 \times 20$ with $N=441$ vertices, when we use $M=4$ multiscale basis functions in each local domain for pressure and similar number of basis functions for temperature, coarse-scale system size is $D O F_{c}=D O F_{c}^{p}=D O F_{c}^{T}=441 \cdot 4=$ 


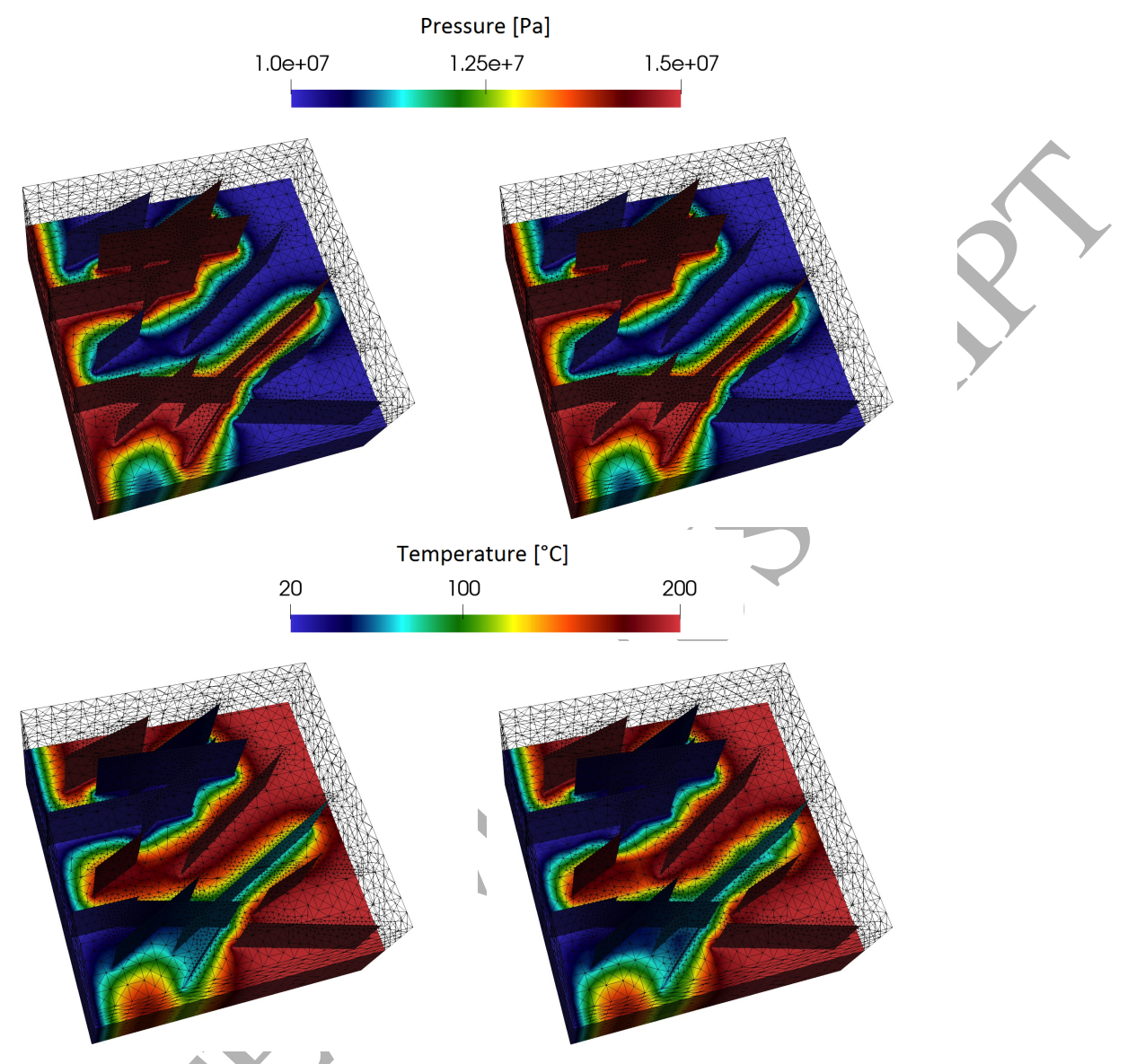

Figure 11: Fine grid and multiscale solution at final time. Three-dimensional problem for Test Case 3. First column: fine grid solution, $D O F_{f}=D O F_{f}^{T}=D O F_{f}^{p}=60$, 206. Second column: multiscale solution, $D O F_{c}=D O F_{c}^{T}=D O F_{c}^{p}=4,356\left(7.2 \%\right.$ from $\left.D O F_{f}\right)$. First row: pressure, $p$. Second row: temperature, $T$. The domain size is $10 \mathrm{~m} \times 10 \mathrm{~m} \times 5 \mathrm{~m}$.

(1762



1762. For the fine-scale system $D O F_{f}=D O F_{f}^{p}=D O F_{f}^{T}=N_{f}=40,395$. Then, we can obtain good solution for multiscale solver for Test Case 2 using only $4.3 \%$ from $D O F_{f}$. For Test Case 3 , for $M=6$ multiscale basis functions on the coarse grid $10 \times 10 \times 5$, we have $D O F_{c}=D O F_{c}^{p}=D O F_{c}^{T}=726 \cdot 6=4,356$ and $D O F_{f}=D O F_{f}^{p}=D O F_{f}^{T}=N_{f}=60,206$. Therefore, for $3 \mathrm{D}$ problem in Test Case 3, we obtain good results using only $7.2 \%$ from $D O F_{f}$. In each time 


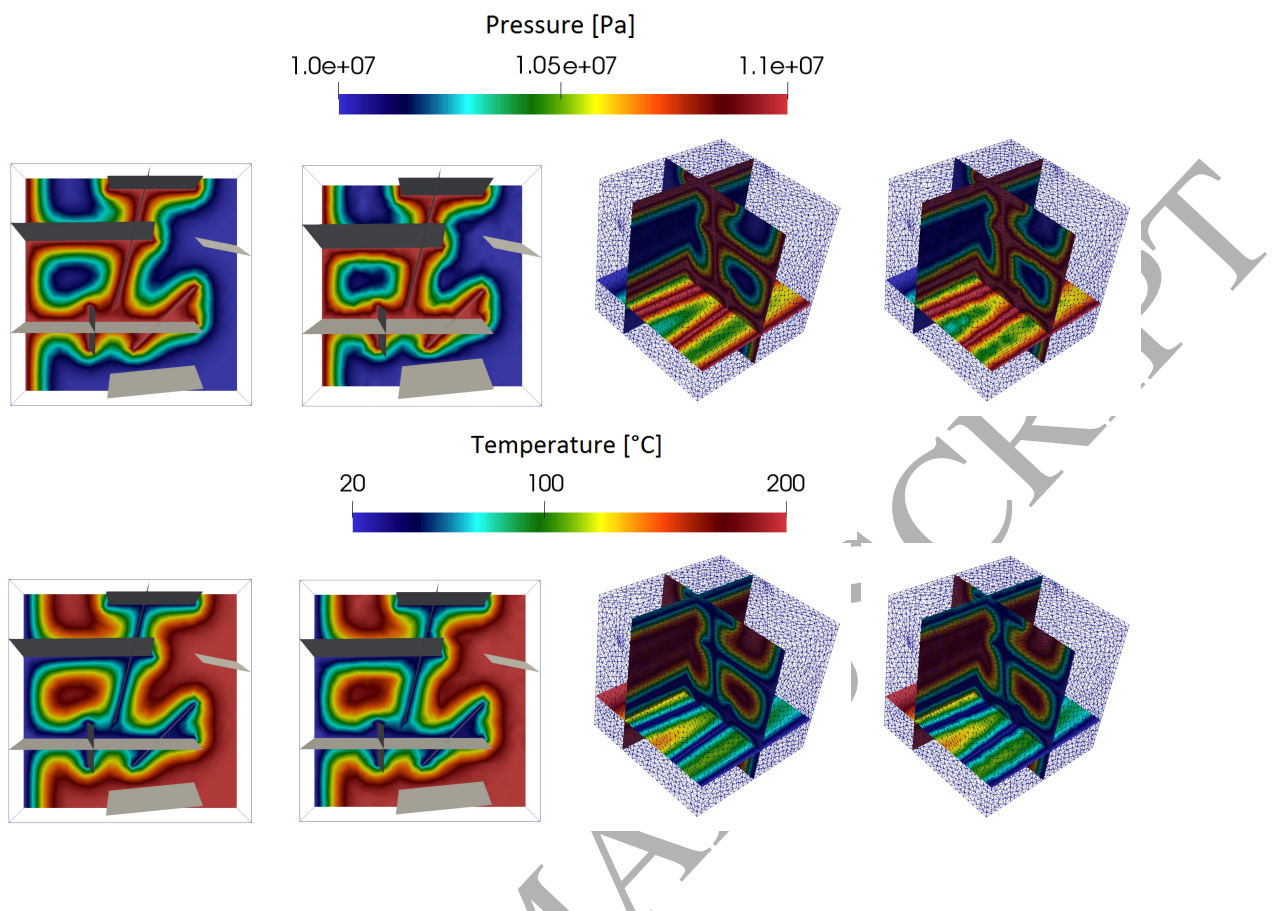

Figure 12: Fine grid and multiscale solution at final time for pressure (first row) and temperature (second row). Three-dimensional problem for Test Case 4. First column: fine grid solution for $Z=5$ slice. Second column: multiscale solution for $Z=5$ slice. Third column: fine grid solution for $X=7, Y=2.5$ and $Z=5$ slices. Fourth column: multiscale solution for $X=7, Y=2.5$ and $Z=5$ slices. The domain size is $10 \mathrm{~m} \times 10 \mathrm{~m} \times 5 \mathrm{~m}$.

step, the proposed method solves a small coarse-grid system compared to the fine-grid system. In both offline and online simulations, one can easily take an advantage of space-time adaptivity and parallel computations.

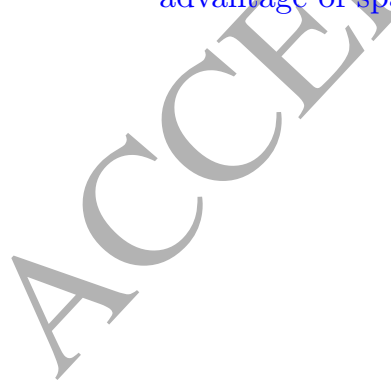




\section{Conclusions and future work}

A Generalized Multiscale Finite Element method was developed successfully for resolving fluid temperature and pressure in fractured porous media. The high temperature gradient is relevant to Enhanced Geothermal Systems (EGS) and therefore the model developed has direct application in design of optimal heat recovery from these geothermal resources. All results showed reasonable accuracy of the proposed multiscale method for heat and mass transfer problem in fractured media relevant to EGS operations.

In the current work, we assumed a small diffusion dominated heat transfer processes. However, the diffusion process may impact the hydrodynamics of mass exchange between fractures and matrix. In the future, we will add mechanical effects and consider coupled thermoporoelastic processes in fractured media. Such numerical applications have been discussed in existing literature studies [52], but not in a computationally efficient multiscale framework. Moreover, in the context of coupled thermo-hydro-mechanical-chemical (THMC) processes, fracture alteration is an area of research for the geothermal heat recovery $[53,54,55,56]$. Computational expenses are always an issue even for modern computers. In future, we explore extending multiscale modelling to these areas of research as well.

\section{Nomenclature}

Mathematical model parameters




$a_{m} \quad$ porosity times coefficient of isothermal compressibility in matrix rock and fluid

$a_{f} \quad$ porosity times coefficient of isothermal compressibility

in fracture fluid

$b \quad$ fracture aperture

$b_{m} \quad$ permeability over viscosity ratio in matrix

$b_{f} \quad$ aperture times permeability divided by viscosity in fracture

$c_{r} \quad$ specific heat of the solid phase

$c_{T}^{w} \quad$ fluid thermal expansion coefficient

$c_{T}^{r} \quad$ rock thermal expansion coefficient

$c_{p}^{w} \quad$ coefficient of isothermal compressibility of fluid

$c_{p}^{r} \quad$ coefficient of isothermal compressibility of rock

$c_{w} \quad$ specific heat of the fluid phase

$h_{m} \quad$ porosity times fluid thermal expansion coefficient in matrix rock and fluid

$h_{f} \quad$ porosity times fluid thermal expansion coefficient in fracture fluid

$L_{f m}$ heat flux between matrix and fracture

$p_{m} \quad$ matrix pressure

$p_{f} \quad$ fracture pressure

$p_{f i} \quad$ fine scale pressure

$q_{m}$ Darcy velocity in matrix

$q_{f}$ average velocity of fluid along the fracture plane

$T_{m} \quad$ matrix temperature

$T_{f} \quad$ fracture temperature

fine scale temperature

$\beta \quad$ porosity averaged fluid and solid thermal conductivity over fracture aperture ratio

$\kappa_{m} \quad$ matrix permeability

$\kappa_{f} \quad$ fracture permeability

$\lambda_{m} \quad$ porosity averaged fluid and solid thermal conductivity in matrix

$\lambda_{f} \quad$ porosity averaged fluid and solid thermal conductivity in fracture 
$\lambda_{r} \quad$ thermal conductivity of rock

$\lambda_{w}$ thermal conductivity of fluid

$\mu \quad$ fluid viscosity

$\rho_{r} \quad$ density of the solid phase

$\rho_{w} \quad$ density of the fluid phase

$\sigma \quad$ matrix-fracture pressure exchange coefficient

$\phi \quad$ porosity

$\phi_{0} \quad$ reference porosity

Fine and coarse grid solvers parameters

$N_{f i} \quad$ number of nodes on the fine grid

DOF Degree of Freedom

$V_{m s} \quad$ multiscale space for pressure

$W_{m s} \quad$ multiscale space for temperature

$V_{\text {snap }}^{\omega} \quad$ snapshot space

$M_{T}^{\omega_{i}} \quad$ number of multiscale basis functions in local domain $\omega_{i}$ for temperature

$M_{p}^{\omega_{i}} \quad$ number of multiscale basis functions in local domain $\omega_{i}$ for pressure

$R \quad$ projection operator for temperature

$P \quad$ projection operator for pressure

$R^{T} \quad$ prolongation operator for temperature

$P^{T} \quad$ prolongation operator for pressure

$\mathcal{T}_{h}$ fine grid

$\mathcal{E}_{h} \quad$ facets of the $\mathcal{T}_{h}$

fracture facets of the $\mathcal{T}_{h}$

standard linear element basis functions

time step

$\omega_{i} \quad$ local domain

$\chi_{i} \quad$ multiscale partition of the unity functions 
$\psi_{k}^{\omega_{i}} \quad$ multiscale basis functions for pressure

$\phi_{k}^{\omega_{i}} \quad$ multiscale basis functions for temperature

$e^{p} \quad L^{2}$ error for pressure

$e^{T} \quad L^{2}$ error for temperature

\section{Acknowledgements}

The authors appreciate constructive comments of the anonymous reviewers who helped improve the quality of paper. MV's and DS's work is supported by the grant of Russian Scientific Foundation N17-71-20055. MB's and EC's contribution to this work is supported by the Chinese University of Hong KongUniversity of Manchester Research Fund 2017. EC's work is partially supported by Hong Kong RGC General Research Fund (Projects: 14317516 and 14304217) and CUHK Direct Grant for Research 2017-18.

\section{References}

\section{References}

[1] O. Ellabban, H. Abu-Rub, F. Blaabjerg, Renewable energy resources: Current status, future prospects and their enabling technology, Renewable and Sustainable Energy Reviews 39 (2014) 748-764.

[2] T. Schulte, G. Zimmermann, F. Vuataz, S. Portier, T. Tischner, R. Junker, R. Jatho, E. Huenges, Enhancing geothermal reservoirs, Geothermal Energy Systems: Exploration, Development, and Utilization (2010) 173-243.

[3] J. W, Tester, B. J. Anderson, A. S. Batchelor, D. D. Blackwell, R. DiPippo, E. M. Drake, J. Garnish, B. Livesay, M. C. Moore, K. Nichols, et al., Impact of enhanced geothermal systems on us energy supply in the twentyfirst century, Philosophical Transactions of the Royal Society of London A: Mathematical, Physical and Engineering Sciences 365 (1853) (2007) 10571094. 
[4] T. C. Ekneligoda, K.-B. Min, Determination of optimum parameters of doublet system in a horizontally fractured geothermal reservoir, Renewable Energy 65 (2014) 152-160.

[5] D. C. Karvounis, Simulations of enhanced geothermal systems with an adaptive hierarchical fracture representation, Ph.D. thesis, ETH Zurich (2013).

[6] D. C. Karvounis, P. Jenny, Adaptive hierarchical fracture model for enhanced geothermal systems, Multiscale Modeling \& Simulation 14 (1) (2016) 207-231.

[7] P. C. Lichtner, Critique of dual continuum formulations of multicomponent reactive transport in fractured porous media, Dynamics of Fluids in Fractured Rock (2000) 281-298.

[8] J. Fairley, S. Ingebritsen, R. Podgorney, Challenges for numerical modeling of enhanced geothermal systems, Groundwater 48 (4) (2010) 482.

[9] Y.-L. He, W.-Q. Tao, Multiscale simulations of heat transfer and fluid flow problems, Journal of Heat Transfer 134 (3) (2012) 031018.

[10] A. Nissen, E. Keilegavlen, T. H. Sandve, I. Berre, J. M. Nordbotten, Heterogeneity preserving upscaling for heat transport in fractured geothermal reservoirs, Computational Geosciences (2017) 1-17.

[11] C. Xu, P. A. Dowd, Z. F. Tian, A simplified coupled hydro-thermal model for enhanced geothermal systems, Applied Energy 140 (2015) 135-145.

[12] J. Geiser, Coupled Systems: Theory, Models, and Applications in Engineering, CRC Press, 2014.

[13] A. Nissen, E. Keilegavlen, T. H. Sandve, I. Berre, J. M. Nordbotten, Heterogeneity preserving upscaling for heat transport in fractured geothermal reservoirs, Computational Geosciences (2017) 1-17. 
[14] T. Praditia, R. Helmig, H. Hajibeygi, Multiscale formulation for coupled flow-heat equations arising from single-phase flow in fractured geothermal reservoirs, Computational Geosciences (2018) 1-18.

[15] Y. Efendiev, J. Galvis, T. Y. Hou, Generalized multiscale finite element methods (gmsfem), Journal of Computational Physics 251 (2013) 116-135.

[16] E. T. Chung, Y. Efendiev, T. Leung, M. Vasilyeva, Coupling of multiscale and multi-continuum approaches, GEM-International Journal on Geomathematics 8 (1) (2017) 9-41.

[17] I. Y. Akkutlu, Y. Efendiev, M. Vasilyeva, Y. Wang, Multiscale model reduction for shale gas transport in a coupled discrete fracture and dualcontinuum porous media, Journal of Natural Gas Science and Engineering.

[18] I. Akkutlu, Y. Efendiev, M. Vasilyeva, Multiscale model reduction for shale gas transport in fractured media, Computational Geosciences (2015) 1-21.

[19] E. Chung, Y. Efendiev, T. Y. Hou, Adáptive multiscale model reduction with generalized multiscale finite element methods, Journal of Computational Physics 320 (2016) 69-95.

[20] V. Girault, K. Kumar, M. F. Wheeler, Convergence of iterative coupling of geomechanics with flow in a fractured poroelastic medium, Computational Geosciences 20 (5) (2016) 997-1011.

[21] V. Martin, J. Jaffré, J. E. Roberts, Modeling fractures and barriers as interfaces for flow in porous media, SIAM Journal on Scientific Computing 26 (5) (2005) 1667-1691.

[22] C. D'Angelo, A. Quarteroni, On the coupling of 1d and 3d diffusion-reaction equations: application to tissue perfusion problems, Mathematical Models and Methods in Applied Sciences 18 (08) (2008) 1481-1504.

[23] L. Formaggia, A. Fumagalli, A. Scotti, P. Ruffo, A reduced model for darcy's problem in networks of fractures, ESAIM: Mathematical Modelling and Numerical Analysis 48 (4) (2014) 1089-1116. 
[24] C. D'Angelo, A. Scotti, A mixed finite element method for darcy flow in fractured porous media with non-matching grids, ESAIM: Mathematical Modelling and Numerical Analysis 46 (2) (2012) 465-489.

[25] V. I. Vasil'ev, A. M. Maksimov, E. E. Petrov, G. G. Tsypkin, Heat and mass transfer in freezing and thawing soils (1996).

[26] N. V. Pavlova, P. N. Vabishchevich, M. V. Vasilyeva, Mathematical modeling of thermal stabilization of vertical wells on high performance computing systems, in: International Conference on Large-Scale Scientific Computing, Springer, 2013, pp. 636-643.

[27] X. Wang, A. Ghassemi, A three-dimensional poŕoelastic model for naturally fractured geothermal reservoir stimulation, in: GRC Annual Meeting. Las Vegas, Nevada, 2013.

[28] C. Rawal, A. Ghassemi, A reactive thermo-poroelastic analysis of water injection into an enhanced geothermal reservoir, Geothermics 50 (2014) $10-23$.

[29] X. Wang, A. Ghassemi, A 3d thermal-poroelastic model for geothermal reservoir stimulation, in: Thirty-seventh workshop on geothermal reservoir engineering, 2012

[30] M. Tene, M. S. Al Kobaisi, H. Hajibeygi, Algebraic multiscale method for flow in heterogeneous porous media with embedded discrete fractures (f-ams), Journal of Computational Physics 321 (2016) 819-845.

[31] M. Tene, M. Al Kobaisi, H. Hajibeygi, Multiscale projection-based embedded discrete fracture modeling approach (f-ams-pedfm), in: ECMOR XV-15th European Conference on the Mathematics of Oil Recovery, 2016.

[32] M. Vasilyeva, E. T. Chung, W. T. Leung, V. Alekseev, Nonlocal multicontinuum (nlmc) upscaling of mixed dimensional coupled flow problem for embedded and discrete fracture models, arXiv preprint arXiv:1805.09407. 
[33] M. Vasilyeva, E. T. Chung, Y. Efendiev, J. Kim, Constrained energy minimization based upscaling for coupled flow and mechanics, arXiv preprint arXiv:1805.09382.

[34] M. Vasilyeva, E. T. Chung, S. W. Cheung, Y. Wang, G. Prokopev, Nonlocal multicontinua upscaling for multicontinua flow problems in fractured porous media, arXiv preprint arXiv:1807.05656.

[35] M. Karimi-Fard, L. J. Durlofsky, K. Aziz, et al., An efficient discrete fracture model applicable for general purpose reservoir simulators, in: SPE Reservoir Simulation Symposium, Society of Petroleum Engineers, 2003.

[36] M. Karimi-Fard, A. Firoozabadi, et al., Numerical simulation of water injection in $2 \mathrm{~d}$ fractured media using discrete-fracture model, in: SPE annual technical conference and exhibition, Society of Petroleum Engineers, 2001.

[37] Y. Efendiev, J. Galvis, G. Li, M. Presho, Generalized multiscale finite element methods. nonlinear elliptic equations, Commun. Comput. Phys. 15(3) (2014) 733-755.

[38] Y. Efendiev, T. Hou, Multiscale Finite Element Methods: Theory and Applications, Vol. 4 of Surveys and Tutorials in the Applied Mathematical Sciences, Springer, New York, 2009.

[39] E. T. Chung, Y. Efendiev, G. Li, M. Vasilyeva, Generalized multiscale finite element method for problems in perforated heterogeneous domains, Applicable Analysis 255 (2015) 1-15.

[40] E. T. Chung, Y. Efendiev, W. T. Leung, Residual-driven online generalized multiscale finite element methods, arXiv preprint arXiv:1501.04565.

[41] E. T. Chung, Y. Efendiev, S. Fu, Generalized multiscale finite element method for elasticity equations, GEM-International Journal on Geomathematics 5 (2) (2014) 225-254. 
[42] E. T. Chung, Y. Efendiev, C. S. Lee, Mixed generalized multiscale finite element methods and applications, Multiscale Modeling \& Simulation 13 (1) (2015) 338-366.

[43] I. Y. Akkutlu, Y. Efendiev, M. Vasilyeva, Multiscale model reduction for shale gas transport in fractured media, Computational Geosciences 20 (5) (2016) 953-973.

[44] Y. Efendiev, S. Lee, G. Li, J. Yao, N. Zhang, Hierarchical multiscale modeling for flows in fractured media using generalized multiscale finite element method, arXiv preprint arXiv:1502.03828To appear in International Journal on Geomathematics, (DOI) 10.1007/s13137-015-0075-7.

[45] E. T. Chung, Y. Efendiev, M. Vasilyeva, Y. Wang, A multiscale discontinuous galerkin method in perforated domains, in: PROCEEDINGS OF THE INSTITUTE OF MATHEMATICS AND MECHANICS, Vol. 42, INST MATHEMATICS \& MECHANICS, NATL ACAD SCIENCES AZERBAIJAN 9 B VAHABZADEH/STR, AZ1141, BAKU, 00000, AZERBAIJAN, 2016, pp. 212-229.

[46] S. Bosma, H. Hajibeygi, M. Tene, H. A. Tchelepi, Multiscale finite volume method for discretefracture modeling on unstructured grids (ms-dfm), Journal of Computational Physics 351 (2017) 145-164.

[47] H. Hajibeygi, G. Bonfigli, M. A. Hesse, P. Jenny, Iterative multiscale finitevolume method, Journal of Computational Physics 227 (19) (2008) 86048621.

[48] J. Galvis, E. Chung, Y. Efendiev, W. T. Leung, On overlapping domain decomposition methods for high-contrast multiscale problems, arXiv preprint arXiv:1705.09004.

[49] J. Galvis, Y. Efendiev, Domain decomposition preconditioners for multiscale flows in high-contrast media, Multiscale Modeling \& Simulation 8 (4) (2010) 1461-1483. 
[50] A. Logg, Efficient representation of computational meshes, International Journal of Computational Science and Engineering 4 (4) (2009) 283-295.

[51] A. Logg, K.-A. Mardal, G. Wells, Automated solution of differential equations by the finite element method: The FEniCS book, Vol. 84, Springer Science \& Business Media, 2012.

[52] H. S. Vik, S. Salimzadeh, H. M. Nick, Heat recovery from multiple-fracture enhanced geothermal systems: The effect of thermoelastic fracture interactions, Renewable Energy 121 (2018) $606-622$.

[53] K. Bisdom, G. Bertotti, H. M. Nick, A geometrically based method for predicting stress-induced fracture aperture and flow in discrete fracture networks, AAPG Bulletin 100 (7) (2016) 1075-1097.

[54] K. Bisdom, H. Nick, G. Bertotti, An integrated workflow for stress and flow modelling using outcrop-derived discrete fracture networks, Computers \& Geosciences 103 (2017) 21-35.

[55] S. Pandey, A. Chaudhuri, S. Kelkar, A coupled thermo-hydro-mechanical modeling of fracture aperture alteration and reservoir deformation during heat extraction from a geothermal reservoir, Geothermics 65 (2017) 17 31.

[56] S. Salimzadeh, A. Paluszny, H. M. Nick, R. W. Zimmerman, A threedimensional coupled thermo-hydro-mechanical model for deformable fractured geothermal systems, Geothermics 71 (2018) $212-224$. 